\title{
A characterisation of ordered abstract probabilities
}

\author{
Abraham Westerbaan \\ Radboud Universiteit \\ Nijmegen \\ bram@westerbaan.name
}

\author{
Bas Westerbaan \\ University College London \\ bas@westerbaan.name
}

\author{
John van de Wetering \\ Radboud Universiteit \\ Nijmegen \\ john@vdwetering.name
}

\begin{abstract}
In computer science, especially when dealing with quantum computing or other non-standard models of computation, basic notions in probability theory like "a predicate" vary wildly. There seems to be one constant: the only useful example of an algebra of probabilities is the real unit interval. In this paper we try to explain this phenomenon. We will show that the structure of the real unit interval naturally arises from a few reasonable assumptions. We do this by studying effect monoids, an abstraction of the algebraic structure of the real unit interval: it has an addition $x+y$ which is only defined when $x+y \leq 1$ and an involution $x \mapsto 1-x$ which make it an effect algebra, in combination with an associative (possibly non-commutative) multiplication. Examples include the unit intervals of ordered rings and Boolean algebras.

We present a structure theory for effect monoids that are $\omega$-complete, i.e. where every increasing sequence has a supremum.

We show that any $\omega$-complete effect monoid embeds into the direct sum of a Boolean algebra and the unit interval of a commutative unital $\mathrm{C}^{*}$-algebra. This gives us from first principles a dichotomy between sharp logic, represented by the Boolean algebra part of the effect monoid, and probabilistic logic, represented by the commutative $\mathrm{C}^{*}$-algebra. Some consequences of this characterisation are that the multiplication must always be commutative, and that the unique $\omega$-complete effect monoid without zero divisors and more than 2 elements must be the real unit interval. Our results give an algebraic characterisation and motivation for why any physical or logical theory would represent probabilities by real numbers.
\end{abstract}

CCS Concepts: • Theory of computation $\rightarrow$ Quantum computation theory; Probabilistic computation.

\footnotetext{
Permission to make digital or hard copies of all or part of this work for personal or classroom use is granted without fee provided that copies are not made or distributed for profit or commercial advantage and that copies bear this notice and the full citation on the first page. Copyrights for components of this work owned by others than the author(s) must be honored. Abstracting with credit is permitted. To copy otherwise, or republish, to post on servers or to redistribute to lists, requires prior specific permission and/or a fee. Request permissions from permissions@acm.org. LICS '20, July 8-11, 2020, Saarbrücken, Germany

(c) 2020 Copyright held by the owner/author(s). Publication rights licensed to ACM.

ACM ISBN 978-1-4503-7104-9/20/07 ..\$15.00

https://doi.org/10.1145/3373718.3394742
}

Keywords: effect monoids, effectus theory, abstract probabilities

\begin{abstract}
ACM Reference Format:
Abraham Westerbaan, Bas Westerbaan, and John van de Wetering. 2020. A characterisation of ordered abstract probabilities. In Proceedings of the 35th Annual ACM/IEEE Symposium on Logic in Computer Science (LICS '20), July 8-11, 2020, Saarbrücken, Germany. ACM, New York, NY, USA, 14 pages. https://doi.org/10.1145/ 3373718.3394742
\end{abstract}

\section{Introduction}

Probability theory in the quantum realm is different in important ways from that of the classical world. Nevertheless, they both crucially rely on real numbers to represent probabilities of events. This makes sense as observations of quantum systems must still be interpreted trough classical means. However, in principle one can imagine a world governed by different physical laws where even the standard notion of a probability is different, or wish to study probabilistic models where one does not care about the specifics of their probabilities; such an approach can for instance be found in categorical quantum mechanics $[1,17,46]$. In this paper we study a reasonable class of alternatives to the real unit interval as the set of allowed probabilities. We will establish that this quite general seeming class actually only contains (continuous products of) the real unit interval. This shows that any 'reasonable' enough physical theory must necessarily be based on probabilities represented by real numbers.

To determine the right set of alternatives to the real unit interval we must first determine which structure is crucial for abstract probabilities. There are a variety of operations on the real unit interval that are used in their interpretation as probabilities. First, the sum $x+y$ of two probabilities $x, y \in[0,1]$ satisfying $x+y \leq 1$ is needed for coarse-graining probabilities of mutually exclusive events. This operation is commutative and associative. Second, to represent the complement of an event we require the involution given by $x^{\perp} \equiv 1-x$. The probability $x^{\perp}$ is the unique number such that $x+x^{\perp}=1$. Axiomatising this structure of a partially defined addition together with an involution defines an effect algebra [12]. Third, in order to talk about, for instance, joint distributions, the multiplication $x \cdot y$ on the unit interval is needed. The multiplication is associative and commutative, which is needed for its relation with joint distributions to hold. Additionally, the multiplication distributes over the addition, which expresses how a joint distribution interacts 
with coarse-grained events. We will not require commutativity of the multiplication as it is not needed for our results. We have then arrived at the notion of an effect monoid: an effect algebra with an associative distributive (possibly noncommutative) multiplication [27]. Effect monoids hence axiomatise the three interacting algebraic structures (addition, involution and multiplication) present in the unit interval.

In order to define an analogue to Bayes' theorem we would also need the division operation that is available in the unit interval: when $x \leq y$, then there is a probability $z$ such that $y \cdot z=x$ (namely $z=x / y$ ). We actually will not require the existence of such a division operation, as it turns out to follow (non-trivially) from our final requirement.

A property that sets the unit interval $[0,1]$ apart from, for instance, the rational numbers between 0 and 1 , is that $[0,1]$ is closed under taking limits, which is indeed a natural property to require. For abstract effect monoids it is however not immediately clear how to express this property, as there is no inherent metric. We propose instead to use the related property of $\omega$-completeness of the order. This says that each ascending chain of elements $x_{1} \leq x_{2} \leq \ldots$ has a supremum. This is a weaker version of directed completeness, which requires the existence of suprema of all directed sets, a property commonly used in computer science. We have then arrived at our candidate for an abstract notion of the set of probabilities: an $\omega$-complete effect monoid.

Further motivation for the use of this structure as a natural candidate for the set of probabilities is its prevalence in effectus theory. This is a recent approach to categorical logic [8] and a general framework to deal with notions such as states, predicates, measurement and probability in deterministic, (classical) probabilistic and quantum settings [7, 55]. The set of probabilities in an effectus have the structure of an effect monoid. Examples of effectuses include any generalised probabilistic theory [4], where the probabilities are the unit interval, but also any topos (and in fact any extensive category with final object), where the probabilities are the Boolean values $\{0,1\}[7]$.

An effectus defines the sum of some morphisms. In a $\sigma$ effectus, this is strengthened to the existence of some countable sums (making it a partially additive category [3]). In such an effectus the probabilities form an $\omega$-complete effect monoid [7].

Effect monoids are of broader interest than only to study effectuses: examples of effect monoids include all Boolean algebras and unit intervals of partially ordered rings. Furthermore any effect monoid can be used to define a generalised notion of convex set and convex effect algebra (by replacing the usual unit interval by elements of the effect monoid, see $[55, \S 179 \& \S 192]$ or [27]).

This now raises the question of how close a probability theory based on an $\omega$-complete effect monoid is to regular probability theory.
Our main result is that $\omega$-complete effect monoids always embed into a direct sum of an $\omega$-complete Boolean algebra and the unit interval of a bounded- $\omega$-complete commutative unital $\mathrm{C}^{*}$-algebra. The latter is isomorphic to $C(X)$ for some basically disconnected compact Hausdorff space $X$. If the effect monoid is directed complete, then it is even isomorphic to the direct sum of a complete Boolean algebra and the unit interval of a monotone complete commutative $\mathrm{C}^{*}$-algebra.

This result basically states that any $\omega$-complete effect monoid can be split up into a sharp part (the Boolean algebra), and a convex probabilistic part (the commutative $\mathrm{C}^{*}$-algebra). This then gives us from basic algebraic and order-theoretic considerations a dichotomy between sharp and fuzzy logic.

As part of the proof of this embedding theorem we find an assortment of additional structure present in $\omega$-complete effect monoids: it has a partially defined division operation, it is a lattice, and multiplication must necessarily be normal (i.e. preserve suprema).

The classification also has some further non-trivial consequences. In particular, it shows that any $\omega$-complete effect monoid must necessarily be commutative.

Finally, we use the classification to show that an $\omega$-complete effect monoid without zero divisors must either be trivial, $\{0\}$, the two-element Boolean algebra, $\{0,1\}$, or the unit interval, $[0,1]$. This gives a new characterisation of the real unit interval as the unique $\omega$-complete effect monoid without zero divisors and more than two elements, and could be seen as a generalisation of the well-known result that the set of real numbers is the unique Dedekind-complete Archimedean ordered field.

In so far as the structure of an $\omega$-complete effect monoid is required for common actions involving probabilities, (coarsegraining, negations, joint distributions, limits) our results motivate the usage of real numbers in any hypothetical alternative physical theory.

\section{Preliminaries}

Before we state the main results of this paper technically, we recall the definitions of the structures involved.

Definition 1. An effect algebra (EA) $\left(E, \emptyset, 0,()^{\perp}\right)$ is a set $E$ with distinguished element $0 \in E$, partial binary operation $\emptyset$ (called sum) and (total) unary operation $x \mapsto x^{\perp}$ (called complement), satisfying the following axioms, writing $x \perp y$ whenever $x \otimes y$ is defined and $1 \equiv 0^{\perp}$.

- Commutativity: if $x \perp y$, then $y \perp x$ and $x \oslash y=y \bowtie x$.

- Zero: $x \perp 0$ and $x \otimes 0=x$.

- Associativity: if $x \perp y$ and $(x \otimes y) \perp z$, then $y \perp z$, $x \perp(y \otimes z)$, and $(x \otimes y) \otimes z=x \otimes(y \otimes z)$.

- For any $x \in E$, the complement $x^{\perp}$ is the unique element with $x \otimes x^{\perp}=1$.

- If $x \perp 1$ for some $x \in E$, then $x=0$. 
For $x, y \in E$ we write $x \leq y$ whenever there is a $z \in E$ with $x \oslash z=y$. This turns $E$ into a poset with minimum 0 and maximum 1. The map $x \mapsto x^{\perp}$ is an order anti-isomorphism. Furthermore $x \perp y$ if and only if $x \leq y^{\perp}$. If $x \leq y$, then the element $z$ with $x \oslash z=y$ is unique and is denoted by $y \ominus x$ [12].

A morphism $f: E \rightarrow F$ between effect algebras is a map such that $f(1)=1$ and $f(x) \perp f(y)$ whenever $x \perp y$, and then $f(x \otimes y)=f(x) \otimes f(y)$. A morphism necessarily preserves the complement, $f\left(x^{\perp}\right)=f(x)^{\perp}$, and the order: $x \leq y \Longrightarrow f(x) \leq f(y)$. A morphism is an embedding when it is also order reflecting: if $f(x) \leq f(y)$ then $x \leq y$. Observe that an embedding is automatically injective. We say $E$ and $F$ are isomorphic and write $E \cong F$ when there exists an isomorphism (i.e. a bijective morphism whose inverse is a morphism too) from $E$ to $F$. Note that an isomorphism is the same as a surjective embedding.

Example 2. Let $\left(B, 0,1, \wedge, \vee,()^{\perp}\right)$ be an orthomodular lattice. Then $B$ is an effect algebra with the partial addition defined by $x \perp y \Longleftrightarrow x \wedge y=0$ and in that case $x \otimes y=x \vee y$. The complement, ( $)^{\perp}$, is given by the orthocomplement, ()$^{\perp}$. The lattice order coincides with the effect algebra order (defined above). See e.g. [53, Prop. 27].

Example 3. Let $G$ be an ordered abelian group (such as the self-adjoint part of a $C^{*}$-algebra). Then any interval $[0, u]_{G} \equiv$ $\{a \in G ; 0 \leq a \leq u\}$ where $u$ is a positive element of $G$ forms an effect algebra, with addition given by $a \perp b \Longleftrightarrow a+b \leq$ $u$ and in that case $a \emptyset b=a+b$. The complement is defined by $a^{\perp}=u-a$. The effect algebra order on $[0, u]_{G}$ coincides with the regular order on $G$.

In particular, the set of effects $[0,1]_{C}$ of a unital $C^{*}$-algebra $C$ forms an effect algebra with $a \perp b \Longleftrightarrow a+b \leq 1$, and $a^{\perp}=1-a$.

Effect algebras have been studied extensively (to name a few: $[5,10,13,18,23,32,40])$ and even found surprising applications in quantum contextuality $[42,44]$ and the study of Lebesgue integration [31]. The following remark gives some categorical motivation to the definition of effect algebras.

Remark 4. An effect algebra is a bounded poset: a partially ordered set with a minimal and maximal element. In [38] it is shown that any bounded poset $P$ can be embedded into an orthomodular poset $K(P)$. This is known as the Kalmbach extension [37]. This extends to a functor from the category of bounded posets to the category of orthomodular posets, and this functor is in fact left adjoint to the forgetful functor going in the opposite direction [25]. This adjunction gives rise to the Kalmbach monad on the category of bounded posets. The Eilenberg-Moore category for the Kalmbach monad is isomorphic to the category of effect algebras, and hence effect algebras are in fact algebras over bounded posets [33].

The category of effect algebras is both complete and cocomplete. There is also an algebraic tensor product of effect algebras that makes the category of effect algebras symmetric monoidal [29]. The monoids in the category of effect algebras resulting from this tensor product are called effect monoids, and they can be explicitly defined as follows:

Definition 5. An effect monoid (EM) [27] is an effect algebra $\left(M, \emptyset, 0,^{\perp}, \cdot\right)$ with an additional (total) binary operation $\cdot$, such that the following conditions hold for all $a, b, c \in M$.

- Unit: $a \cdot 1=a=1 \cdot a$.

- Distributivity: if $b \perp c$, then $a \cdot b \perp a \cdot c, \quad b \cdot a \perp c \cdot a$, $a \cdot(b \otimes c)=(a \cdot b) \otimes(a \cdot c)$, and $(b \otimes c) \cdot a=(b \cdot a) \emptyset(c \cdot a)$.

Or, in other words, the operation · is bi-additive.

- Associativity: $a \cdot(b \cdot c)=(a \cdot b) \cdot c$.

We call an effect monoid $M$ commutative if $a \cdot b=b \cdot a$ for all $a, b \in M$; an element $p$ of $M$ idempotent whenever $p^{2} \equiv$ $p \cdot p=p$; elements $a, b$ of $M$ orthogonal when $a \cdot b=b \cdot a=0$; and we denote the set of idempotents of $M$ by $P(M)$.

Notation 6. Following the usual binding conventions for addition and multiplication, the expression $a \cdot b \otimes c$ denotes $(a$. b) $\varnothing c$.

Remark 7. Effect monoids were introduced by Jacobs in [2729] and have been studied explicitly by e.g. [2, 7, 8, 30, 35, $53,55,58]$. Two large subclasses of effect monoids have been studied under a different name: commutative sequential effect algebras [21] are the same thing as commutative effect monoids and a distributive sequential effect algebras [24] is the same thing as an effect monoid with $a \cdot b=$ $0 \Leftrightarrow b \cdot a=0$. Especially (commutative) sequential effect algebras have received a lot of study - to name a few: $[16,19,20,39,43,45,47,48,50]$.

Example 8. Any Boolean algebra $\left(B, 0,1, \wedge, \vee,()^{\perp}\right)$, being an orthomodular lattice, is an effect algebra by Example 2, and, moreover, a commutative effect monoid with multiplication defined by $x \cdot y=x \wedge y$. Conversely, any orthomodular lattice for which $\wedge$ distributes over $\emptyset$ (and thus $\vee$ ) is a Boolean algebra.

Example 9. The unit interval $[0,1]_{R}$ of any (partially) ordered unital ring $R$ (in which the sum $a+b$ and product $^{1} a \cdot b$ of positive elements $a$ and $b$ are again positive) is an effect monoid.

Let, for example, $X$ be a compact Hausdorff space. We denote its space of continuous functions into the complex numbers by $C(X) \equiv\{f: X \rightarrow \mathbb{C}$, continuous $\}$. This is a commutative unital $C^{*}$-algebra (and conversely by the Gel'fand theorem, any commutative $\mathrm{C}^{*}$-algebra with unit is of this form) and hence its unit interval $[0,1]_{C(X)}$ consisting of continuous $f: X \rightarrow[0,1]$ is a commutative effect monoid.

\footnotetext{
${ }^{1}$ There are many interesting rings (like non-commutative $C^{*}$-algebras) whose additive group is ordered, but where the product of positive elements need not be positive. The unit intervals of those rings are not effect monoids.
} 
The unit interval of an ordered ring does not have to be commutative; in [7, Ex. 4.3.9] and [53, Cor. 51] two different non-commutative effect monoids are constructed.

Definition 10. Let $M$ and $N$ be effect monoids. A morphism from $M$ to $N$ is a morphism of effect algebras with the added condition that $f(a \cdot b)=f(a) \cdot f(b)$ for all $a, b \in M$. Similar to the case of effect algebras, an embedding $M \rightarrow N$ is a morphism that is order reflecting. Also here an isomorphism of effect monoids is the same thing as a surjective embedding of effect monoids.

Example 11. It is well-known that any Boolean algebra $B$ is isomorphic to the set of clopens of its Stone space $X_{B}$ [34]. This yields an effect monoid embedding from $B$ into $[0,1]_{C}\left(X_{B}\right)$.

Remark 12. A physical or logical theory which has probabilities of the form $[0,1]_{C(X)}$ can be seen as a theory with a natural notion of space, where probabilities are allowed to vary continuously over the space $X$. Such a spatial theory is considered in for instance Ref. [11].

Example 13. Given two effect algebras/monoids $E_{1}$ and $E_{2}$ we define their direct sum $E_{1} \oplus E_{2}$ as the Cartesian product with pointwise operations. This is again an effect algebra/monoid.

Example 14. Let $M$ be an effect monoid and let $p \in M$ be some idempotent. The subset $p M \equiv\{p \cdot a ; a \in M\}$ is called the left corner by $p$ and is an effect monoid with $(p \cdot a)^{\perp} \equiv$ $p \cdot a^{\perp}$ and all other operations inherited from $M$. Later we will see that $a \mapsto\left(p \cdot a, p^{\perp} \cdot a\right)$ is an isomorphism $M \cong p M \oplus p^{\perp} M$ Analogous facts hold for the right corner $M p \equiv\{a \cdot p ; a \in$ $M\}$.

Definition 15. Let $E$ be an effect algebra. A directed set $S \subseteq E$ is a non-empty set such that for all $a, b \in S$ there exists a $c \in S$ such that $a, b \leq c$. $E$ is directed complete when for any directed set $S$ there is a supremum $\bigvee S$. It is $\omega$-complete if directed suprema of countable sets exist, or equivalently if any increasing sequence $a_{1} \leq a_{2} \leq \ldots$ in $E$ has a supremum.

Remark 16. A directed complete partially ordered set is often referred to by the shorthand dcpo. These structures lie at the basis of domain theory and are often encountered when studying denotational semantics of programming languages as they allow for a natural way to talk about fix points of recursion. Note that being $\omega$-complete is strictly weaker. For effect algebras we could have equivalently defined directed completeness with respect to downwards directed sets, as the complement is an order anti-isomorphism.

Example 17. Let $B$ be a $\omega$-complete Boolean algebra. Then $B$ is a $\omega$-complete effect monoid. If $B$ is complete as a Boolean algebra, then $B$ is directed-complete as effect monoid.

Example 18. Let $X$ be an extremally disconnected compact Hausdorff space, i.e. where the closure of every open set is open. Then $[0,1]_{C(X)}$ is a directed-complete effect monoid. If $X$ is a basically disconnected $[15,1 \mathrm{H}]$ compact Hausdorff space, i.e. where every cozero set has open closure, then $[0,1]_{C(X)}$ is an $\omega$-complete effect monoid [15, 3N.5].

\section{Overview}

The main results of the paper are the following theorems:

Theorem. Let $M$ be an $\omega$-complete effect monoid. Then $M$ embeds into $M_{1} \oplus M_{2}$, where $M_{1}$ is an $\omega$-complete Boolean algebra, and $M_{2}=[0,1]_{C(X)}$, where $X$ is a basically disconnected compact Hausdorff space (see Theorem 70).

Theorem. Let $M$ be a directed-complete effect monoid. Then $M \cong M_{1} \oplus M_{2}$ where $M_{1}$ is a complete Boolean algebra and $M_{2}=[0,1]_{C(X)}$ for some extremally-disconnected compact Hausdorff space $X$ (see Theorem 71).

By Example 11, the Boolean algebra $M_{1}$ also embeds into

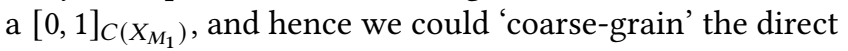
sums above and say that any $\omega$-complete effect monoid embeds into the unit interval of $C\left(X_{M_{1}}+X\right)$, where + is the disjoint union of the topological spaces. This observation suggests a Stone-type duality that we discuss in more detail in the conclusion.

Other results for an $\omega$-complete effect monoid $M$ that either follow directly from the above theorems, or are proven along the way are the following:

- $M$ is a lattice.

- $M$ is an effect divisoid [55, §195].

- The multiplication in $M$ is normal: $a \cdot \bigvee S=\bigvee a \cdot S$.

- If $M$ is convex (as an effect algebra), then scalar multiplication is homogeneous: $\lambda(a \cdot b)=(\lambda a) \cdot b=a \cdot(\lambda b)$ for any $\lambda \in[0,1]$ and $a, b \in M$.

- $M$ is commutative.

- If $M$ has no non-trivial zero-divisors (i.e. $a \cdot b=0$, implies $a=0$ or $b=0)$, then $M$ is isomorphic to $[0,1]$, $\{0,1\}$ or $\{0\}$.

It should be noted that the scalars in a $\sigma$-effectus satisfying normalisation have no non-trivial zero-divisors [7] and hence using the last point above, we have completely characterised the scalars in such $\sigma$-effectuses, splitting them up into trivial, Boolean and convex effectuses. For the details we refer to the follow-up work [9].

The paper is structured as follows. In Section 4 we recover and prove some basic results regarding effect algebras/monoids. Then in Section 5 we will show that in any $\omega$-complete effect monoid $M$, we can define a kind of partial division operation which turns it into a effect divisoid. Using this division we show that the multiplication must be normal. Then in Section 6 we study idempotents that are either Boolean, meaning that all elements below $p$ must also be idempotents, or halvable, meaning that there is an $a \in M$ such that $p=a \oslash a$. We establish that an $\omega$-complete effect monoid where 1 is Boolean must be a Boolean algebra, while 
if 1 is halvable then it must be convex. In Section 7 we show that a maximal collection of orthogonal idempotents of $M$ can be found that consists of a mix of halvable and Boolean idempotents. The corner $p M$ associated to such an idempotent will either be convex (if $p$ is halvable) or Boolean (if $p$ is Boolean). Using normality of multiplication we show that $M$ embeds into the direct sum of the corners associated to these idempotents. Letting $M_{1}$ be the direct sum of the Boolean corners, and $M_{2}$ be the direct sum of the convex corners, we see that $M$ embeds into $M_{1} \oplus M_{2}$, where $M_{1}$ is Boolean and $M_{2}$ is convex. In Section 8, we recall some results regarding order unit spaces and use Yosida's representation theorem to show that a convex $\omega$-complete effect monoid must be isomorphic to the unit interval of a $C(X)$. Then in Section 9 we collect all the results and prove our main theorems. Finally in Section 10 we conclude and discuss some future work and open questions.

\section{Basic results}

We do not assume any commutativity of the product in an effect monoid. Nevertheless, some commutativity comes for free.

Lemma 19. For any $a \in M$ in an effect monoid $M$, we have $a \cdot a^{\perp}=a^{\perp} \cdot a$.

Proof. Using distributivity $a^{2} \otimes\left(a^{\perp} \cdot a\right)=\left(a \otimes a^{\perp}\right) \cdot a=$ $1 \cdot a=a=a \cdot 1=a \cdot\left(a \oslash a^{\perp}\right)=a^{2} \emptyset\left(a \cdot a^{\perp}\right)$. Cancelling $a^{2}$ on both sides gives the desired equality.

Lemma 20. An element $p \in M$ is an idempotent if and only if $p \cdot p^{\perp}=0$.

Proof. $p=p \cdot 1=p \cdot\left(p \otimes p^{\perp}\right)=p^{2} \oslash p \cdot p^{\perp}$. Hence $p=p^{2}$ if and only if $p \cdot p^{\perp}=0$.

Lemma 21. For $a, p \in M$ with $p^{2}=p$, we have

$$
p \cdot a=a \Longleftrightarrow a \cdot p=a \quad \Longleftrightarrow \quad a \leq p .
$$

Proof. Assume $a \leq p$. Then $a \cdot p^{\perp} \leq p \cdot p^{\perp}=0$, so that $a \cdot p^{\perp}=$ 0 . Similarly $p^{\perp} \cdot a=0$. Hence $a=a \cdot 1=a \cdot\left(p \oslash p^{\perp}\right)=$ $a \cdot p \otimes a \cdot p^{\perp}=a \cdot p$. Similarly $p \cdot a=a$.

Now assume $p \cdot a=a$. Then immediately $a=p \cdot a \leq p \cdot 1=$ $p$. The final implication (that $a \cdot p=a \Longrightarrow a \leq p$ ) is proven similarly.

Lemma 22. Let $M$ be an effect monoid with idempotent $p \in$ $M$. Then $p \cdot a=a \cdot p$ for any $a \in M$.

Proof. Clearly $p \cdot a \leq p \cdot 1=p$ and so by Lemma $21 p \cdot a \cdot p=p \cdot a$. Similarly $a \cdot p \leq p$ and so $p \cdot a \cdot p=a \cdot p$. Thus $p \cdot a=p \cdot a \cdot p=a \cdot p$, as desired.

Corollary 23. Let $M$ be an effect monoid with idempotent $p \in M$. The map $e \mapsto\left(p \cdot e, p^{\perp} \cdot e\right)$ is an isomorphism $M \cong$ $p M \oplus p^{\perp} M$.

The following two lemmas are simple observations that will be used several times.
Lemma 24. Let $a \leq b$ be elements of an effect algebra $E$. If $b \otimes b^{\prime} \leq a \otimes a^{\prime}$ for some $a^{\prime} \leq b^{\prime}$ from $E$, then $a=b$ (and $a^{\prime}=b^{\prime}$ ).

Proof. Since $a \leq b$ and $a^{\prime} \leq b^{\prime}$, we have $a \otimes a^{\prime} \leq b \otimes b^{\prime}$, and so $a \otimes a^{\prime}=b \otimes b^{\prime}$. Then $0=\left(b \otimes b^{\prime}\right) \ominus\left(a \otimes a^{\prime}\right)=$ $(b \ominus a) \emptyset\left(b^{\prime} \ominus a^{\prime}\right)$, yielding $b \ominus a=0$ and $b^{\prime} \ominus a^{\prime}=0$, so $b=a$ and $b^{\prime}=a^{\prime}$.

Lemma 25. Let $p$ be an idempotent from an effect monoid $M$, and let $a, b \leq p$ be elements below $p$. If $a \emptyset b$ exists, then $a \emptyset$ $b \leq p$.

Proof. Since $a \leq p$, we have $a \cdot p^{\perp}=0$, and similarly, $b \cdot p^{\perp}=0$. But then $(a \otimes b) \cdot p^{\perp}=0$, and hence $(a \otimes b) \cdot p=a \otimes b$. By Lemma 21 we then have $a \emptyset b \leq p$.

We defined directed set to mean upwards directed. Using the fact that $a \mapsto a^{\perp}$ is an order anti-isomorphism, a directedcomplete effect algebra also has all infima of downwards directed (or 'filtered') sets (and similarly for countable infima in a $\omega$-complete effect algebra).

Recall that given an element $a$ of an ordered group $G$ a subset $S$ of $G$ has a supremum $\bigvee S$ in $G$ if and only if $\bigvee_{s \in S} a+$ $s$ exists, which follows immediately from the observation that $a+(): G \rightarrow G$ is an order isomorphism. For effect algebras the situation is a bit more complicated, and we only have the implications mentioned in the lemma below. We will see in Corollary 41 that the situation improves somewhat for $\omega$-complete effect monoids.

Lemma 26. Let $x$ be an element and $S$ a non-empty subset of an effect algebra $E$. If $S \subseteq\left[0, x^{\perp}\right]_{E}$, then

$$
\begin{aligned}
\bigvee S \text { exists } & \Longrightarrow & x \otimes \bigvee S & =\bigvee x \otimes S, \text { and } \\
\bigwedge x \otimes S \text { exists } & \Longrightarrow & x \otimes \bigwedge S & =\bigwedge x \otimes S .
\end{aligned}
$$

Here "=" means also that the sums, suprema and infima on either side exist. Similarly, if $S \subseteq[x, 1]_{E}$, then

$$
\begin{aligned}
\bigwedge_{s \in S} s \ominus x \text { exists } & \Longrightarrow(\bigvee S) \ominus x=\bigvee_{s \in S} s \ominus x, \text { and } \\
\bigwedge S \text { exists } & \Longrightarrow(\bigwedge S) \ominus x=\bigwedge_{s \in S} s \ominus x .
\end{aligned}
$$

Moreover, if $S \subseteq[0, x]_{E}$, then

$$
\begin{aligned}
\bigvee S \text { exists } & \Longrightarrow & x \ominus \bigvee S=\bigwedge x \ominus S, \text { and } \\
\bigvee x \ominus S \text { exists } & \Longrightarrow & x \ominus \bigwedge S=\bigvee x \ominus S .
\end{aligned}
$$

Proof. Note that $a \mapsto x \otimes a$ gives an order isomorphism $\left[0, x^{\perp}\right]_{E} \rightarrow[x, 1]_{E}$ with inverse $a \mapsto a \ominus x$. Whence $x \emptyset$ ( ) preserves and reflects all infima and suprema restricted to $\left[0, x^{\perp}\right]_{E}$ and $[x, 1]_{E}$. Surely, given elements $a \leq b$ from $E$, and a subset $S$ of the interval $[a, b]_{E}$, it is clear that any supremum (infimum) of $S$ in $E$ will be the supremum (infimum) of $S$ in $[a, b]_{E}$ too (using here that $S$ is non-empty). The converse does not always hold, but when $S$ has a supremum 
in $[a, 1]_{E}$, then this is the supremum in $E$ too (and when $S$ has an infimum in $[0, b]_{E}$, then this is the infimum in $E$ too). These considerations yield the first four equations. For the latter two we just add the observation that $x \ominus$ ( ) gives an order reversing isomorphism $[0, x]_{E} \rightarrow[0, x]_{E}$.

We can now prove a few basic yet useful facts of $\omega$-complete effect monoids. These lemmas deal with elements that are summable with themselves: elements $a$ such that $a \perp a$ which means that $a \oslash a$ is defined. For $n \in \mathbb{N}$ we will use the notation $n a=a \emptyset \ldots \otimes a$ for the $n$-fold sum of $a$ with itself (when it is defined). We study these self-summable elements to be able to define a " $\frac{1}{2}$ " in some effect monoids later on.

Lemma 27. For any $a \in M$ in some effect monoid $M$, the element $a \cdot a^{\perp}$ is summable with itself.

Proof. Since $1=1 \cdot 1=\left(a \emptyset a^{\perp}\right) \cdot\left(a \otimes a^{\perp}\right)=a \cdot a \emptyset a \cdot a^{\perp} \emptyset$ $a^{\perp} \cdot a \otimes a^{\perp} \cdot a^{\perp}$, and $a \cdot a^{\perp}=a^{\perp} \cdot a$ by Lemma 19 , we see that $a \cdot a^{\perp} \oslash a \cdot a^{\perp}$ indeed exists.

Lemma 28. Let $a$ be an element of an $\omega$-complete effect monoid $M$.

1. If $n a$ exists for all $n$ then $a=0$.

2. If $a^{2}=0$ then $a=0$.

3. If $a \perp a$ then $\bigwedge_{n} a^{n}=0$.

Proof. For point 1, we have $a \bigotimes \bigvee_{n} n a=\bigvee_{n} a \emptyset n a=\bigvee_{n}(n+$ 1) $a=\bigvee_{n} n a$, and so $a=0$.

For point 2, since $a^{2}=0$ we have $a=a \cdot 1=a \cdot\left(a \otimes a^{\perp}\right)=$ $a \cdot a^{\perp}$, and hence (because of Lemma 27) $a$ is summable with itself. But furthermore $(a \otimes a)^{2}=4 a^{2}=0$, and so $(a \otimes a)^{2}=0$. Continuing in this fashion, we see that $2^{n} a$ exists for every $n \in \mathbb{N}$ and $\left(2^{n} a\right)^{2}=0$. Hence, for any $m \in \mathbb{N}$ the sum $m a$ exists so that by the previous point $a=0$.

For point 3 , write $b \equiv \bigwedge_{n} a^{n}$. As $(2 a)^{n}=2^{n} a^{n}$ and $b \leq a^{n}$ we see that $2^{n} b$ is defined. But this is true for all $n$, and so again by the point $1, b=0$.

\section{Floors, ceilings and division}

In this section we will see that any $\omega$-complete effect monoid has floors and ceilings. These are respectively the largest idempotent below an element and the smallest idempotent above an element. We will also construct a "division": for $a \leq b$ we will find an element $a / b$ such that $(a / b) \cdot b=a$. These operations have been studied before in the context of von Neumann algebras [51, §56 \& §81].

Then using ceilings and this division we will show that multiplication in a $\omega$-complete effect monoid is always normal, i.e. that $b \cdot \bigvee S=\bigvee b \cdot S$ for non-empty $S$ for which $\bigvee S$ exists. This technical result will be frequently used in the remaining sections.

Definition 29. Let $\left(x_{i}\right)_{i \in I}$ be a (potentially infinite) family of elements from an effect algebra $E$. We say the sum $\nabla_{i \in I} x_{i}$ exists if for every finite subset $S \subseteq I$ the sum $\emptyset_{i \in S} x_{i}$ exists and the supremum $\bigvee_{\text {finite } S \subseteq I} \bigotimes_{i \in S} x_{i}$ exists as well. In that case we write $\emptyset_{i \in I} x_{i} \equiv \bigvee_{\text {finite } S \subseteq I} \emptyset_{i \in S} x_{i}$.

Lemma 30. Given $a \in M$ for an effect monoid $M$, we have

$$
\left(a^{N}\right)^{\perp}=a^{\perp} \oslash a^{\perp} \cdot a \emptyset a^{\perp} \cdot a^{2} \emptyset \cdots \emptyset a^{\perp} \cdot a^{N-1}
$$

for every natural number $N$.

Proof. The result follow immediately from:

$$
\begin{aligned}
1 & =a^{\perp} \oslash a \\
& =a^{\perp} \oslash\left(a^{\perp} \oslash a\right) \cdot a \equiv a^{\perp} \oslash a^{\perp} \cdot a \oslash a^{2} \\
& =a^{\perp} \oslash a^{\perp} \cdot a \oslash a^{\perp} \cdot a^{2} \oslash a^{3} \\
& \cdots=\left(\emptyset_{n=0}^{N-1} a^{\perp} \cdot a^{n}\right) \oslash a^{N} \square
\end{aligned}
$$

Corollary 31. The sum $\emptyset_{n=0}^{\infty} a^{\perp} \cdot a^{n}$ exists for any element $a$ from an $\omega$-complete effect monoid $M$.

Definition 32. Given an element $a$ of an $\omega$-complete effect monoid $M$

$$
\lceil a\rceil \equiv \bigotimes_{n=0}^{\infty} a \cdot\left(a^{\perp}\right)^{n} \quad \text { and } \quad\lfloor a\rfloor \equiv \bigwedge_{n=0}^{\infty} a^{n}
$$

are called the ceiling of a and the floor of $a$, respectively.

We list some basic properties of $\lceil a\rceil$ and $\lfloor a\rfloor$ in Proposition 37, after we have made the observations necessary to establish them.

Lemma 33. Given an element $a$ of an $\omega$-complete effect monoid $M$, we have $\bigwedge_{n} a^{\perp} \cdot a^{n}=0$.

Proof. Write $b \equiv \bigwedge_{n} a^{\perp} \cdot a^{n}$. Since $a$ and $a^{\perp}$ commute by Lemma 19, we compute

$$
1=1^{n}=\left(a^{\perp} \oslash a\right)^{n}=\bigotimes_{k=0}^{n}\left(\begin{array}{l}
n \\
k
\end{array}\right)\left(\left(a^{\perp}\right)^{k} \cdot a^{n-k}\right),
$$

and in particular see that the sum $\left(\begin{array}{c}n \\ 1\end{array}\right)\left(a^{\perp} \cdot a^{n-1}\right) \equiv n\left(a^{\perp} \cdot a^{n-1}\right)$ exists. Because $b \leq a^{\perp} \cdot a^{n-1}$, the $n$-fold sum $n b$ exists too and hence $b=0$ by Lemma 28 .

Lemma 34. We have $\lfloor a\rfloor=\lfloor a\rfloor \cdot a=a \cdot\lfloor a\rfloor$ for any element $a$ of an $\omega$-complete effect monoid $M$.

Proof. Using Lemmas 19 and 33 we compute $\lfloor a\rfloor \cdot a^{\perp}=$ $\left(\bigwedge_{n} a^{n}\right) \cdot a^{\perp} \leq \bigwedge_{n} a^{n} \cdot a^{\perp}=\bigwedge_{n} a^{\perp} \cdot a^{n}=0$, and so $\lfloor a\rfloor \cdot a=\lfloor a\rfloor$. The other identity has a similar proof.

Lemma 35. Given elements $a, b_{1}, b_{2}, \ldots$ of a $\omega$-complete effect monoid $M$ such that $\emptyset_{n} b_{n}$ exists, and $a \cdot b_{n}=0$ for all $n \in \mathbb{N}$, we have $a \cdot \emptyset_{n} b_{n}=0$.

Proof. Writing $s_{N} \equiv \emptyset_{n=1}^{N} b_{n}$, we have $s_{1} \leq s_{2} \leq \cdots$ and $a$. $s_{n}=0$ for all $n$. Since $s_{n}=\left(a \oslash a^{\perp}\right) \cdot s_{n}=a \cdot s_{n} \oslash a^{\perp} \cdot s_{n}=a^{\perp} \cdot s_{n}$ for all $n \in \mathbb{N}$, we have

$$
\bigvee_{n} s_{n}=\bigvee_{n} a^{\perp} \cdot s_{n} \leq a^{\perp} \cdot \bigvee_{n} s_{n} \leq \bigvee_{n} s_{n}
$$

which implies that $a^{\perp} \cdot \bigvee_{n} s_{n}=\bigvee_{n} s_{n}$, and thus $a \cdot \emptyset_{n} b_{n} \equiv$ $a \cdot \bigvee_{n} s_{n}=0$. 
Proposition 36. Given elements $a$ and $b$ of an $\omega$-complete effect monoid $M, a \cdot b=0 \Longrightarrow a \cdot\lceil b\rceil=0$.

Proof. If $a \cdot b=0$, then also $a \cdot b \cdot\left(b^{\perp}\right)^{n}=0$ for all $n$. Hence by Lemma $35 a \cdot\lceil b\rceil \equiv a \cdot \emptyset_{n=1}^{\infty} b \cdot\left(b^{\perp}\right)^{n}=0$.

Proposition 37. Let $a$ be an element of an $\omega$-complete effect monoid $M$.

1. We have $\lceil a\rceil^{\perp}=\left\lfloor a^{\perp}\right\rfloor$ and $\lfloor a\rfloor^{\perp}=\left\lceil a^{\perp}\right\rceil$.

2. The floor $\lfloor a\rfloor$ of $a$ is an idempotent with $\lfloor a\rfloor \leq a$. In fact, $\lfloor a\rfloor$ is the greatest idempotent below $a$.

3. The ceiling $\lceil a\rceil$ of $a$ is the least idempotent above $a$.

Proof. Point 1 follows from Lemma 30. Concerning point 2 : Since $\lfloor a\rfloor \cdot a^{\perp}=0$ (by Lemma 34) we have $\lfloor a\rfloor \cdot\left\lceil a^{\perp}\right\rceil=0$ by Proposition 36, and so $\lfloor a\rfloor \cdot\lfloor a\rfloor^{\perp}=0$ because $\lfloor a\rfloor^{\perp}=\left\lceil a^{\perp}\right\rceil$ by point 1 . Hence $\lfloor a\rfloor$ is an idempotent. Also, since $\lfloor a\rfloor=\bigwedge_{n} a^{n}$, we clearly have $\lfloor a\rfloor \leq a$. Now, if $s$ is an idempotent in $M$ with $s \leq a$, then $s=s^{n} \leq a^{n}$, and so $s \leq \bigwedge_{n} a^{n} \equiv\lfloor a\rfloor$. Whence $\lfloor a\rfloor$ is the greatest idempotent below $a$. Point 3 now follows easily from 2 , since $\lceil\cdot\rceil$ is the dual of $\lfloor\cdot\rfloor$ under the order anti-isomorphism $(\cdot)^{\perp}$.

Lemma 38. $\lceil a \otimes b\rceil=\lceil a\rceil \vee\lceil b\rceil$ for all summable elements $a$ and $b$ of an $\omega$-complete effect monoid $M$ (that is, $\lceil a \otimes b\rceil$ is the supremum of $\lceil a\rceil$ and $\lceil b\rceil$ ).

Proof. Since $\lceil a \otimes b\rceil \geq a \otimes b \geq a$, we have $\lceil a \emptyset b\rceil \geq\lceil a\rceil$, and similarly, $\lceil a \otimes b\rceil \geq\lceil b\rceil$. Let $u$ be an upper bound of $\lceil a\rceil$ and $\lceil b\rceil$; we claim that $\lceil a \boxminus b\rceil \leq u$. Since $\lceil a\rceil \leq u$ and $\lceil b\rceil \leq u$, we have $a \leq\lceil a\rceil \leq\lfloor u\rfloor$ and $b \leq\lceil b\rceil \leq\lfloor u\rfloor$, and so $a \emptyset b \leq$ $\lfloor u\rfloor$ by Lemma 25 . Whence $\lceil a \otimes b\rceil \leq\lfloor u\rfloor \leq u$.

Any $\omega$-complete effect monoid is a lattice effect algebra [41]:

Theorem 39. All elements $a$ and $b$ from an $\omega$-complete effect monoid $M$ have an infimum, $a \wedge b$, given by

$$
a \wedge b=\bigotimes_{n=1}^{\infty} a_{n} \cdot b_{n} \text { where }\left[\begin{array}{ll}
a_{1}=a & a_{n+1}=a_{n} \cdot b_{n}^{\perp} \\
b_{1}=b & b_{n+1}=a_{n}^{\perp} \cdot b_{n}
\end{array} ;\right.
$$

and thus also a supremum, given by $a \vee b=\left(a^{\perp} \wedge b^{\perp}\right)^{\perp}$.

Proof. First we need to show that the sum $\emptyset_{n=1}^{N} a_{n} \cdot b_{n}$ exists for every $N$. In fact, we'll show that $a \ominus \emptyset_{n=1}^{N} a_{n} \cdot b_{n}=a_{N+1}$ for all $N$, by induction. Indeed, for $N=1$, we have $a \ominus a \cdot b=$ $a \cdot b^{\perp}=a_{2}$, and if $a \ominus \emptyset_{n=1}^{N} a_{n} \cdot b_{n}=a_{N+1}$ for some $N$, then $a_{N+2}=a_{N+1} \cdot b_{N+1}^{\perp}=a_{N+1} \ominus a_{N+1} \cdot b_{N+1}=(a \ominus$ $\left.\emptyset_{n=1}^{N} a_{n} \cdot b_{n}\right) \ominus a_{N+1} \cdot b_{N+1}=a \ominus \emptyset_{n=1}^{N+1} a_{n} \cdot b_{n}$. In particular, $\nabla_{n=1}^{\infty} a_{n} \cdot b_{n}$ exists, and, moreover,

$$
a=\bigwedge_{m=1}^{\infty} a_{m} \oslash \emptyset_{n=1}^{\infty} a_{n} \cdot b_{n} .
$$

By a similar reasoning, we get $b=\bigwedge_{m=1}^{\infty} b_{m} \otimes \bigotimes_{n=1}^{\infty} a_{n} \cdot b_{n}$. Already writing $a \wedge b \equiv \emptyset_{n=1}^{\infty} a_{n} \cdot b_{n}$, we know at this point that $a \wedge b \leq a$ and $a \wedge b \leq b$. It remains to be shown that $a \wedge b$ defined above is the greatest lower bound of $a$ and $b$. So let $\ell \in M$ with $\ell \leq a$ and $\ell \leq b$ be given; we must show that $\ell \leq a \wedge b$.
As an intermezzo, we observe that $\left(\bigwedge_{n} a_{n}\right) \cdot\left(\bigwedge_{m} b_{m}\right)=$ 0 . Indeed, we have $\left(\bigwedge_{n} a_{n}\right) \cdot\left(\bigwedge_{m} b_{m}\right) \leq \bigwedge_{n} a_{n} \cdot b_{n}$, and $\bigwedge_{n} a_{n} \cdot b_{n}=0$, because $\emptyset_{n=1}^{\infty} a_{n} \cdot b_{n}$ exists (see Lemma 28). By Proposition 36 it follows that $\left(\bigwedge_{n} a_{n}\right) \cdot\left\lceil\bigwedge_{m} b_{m}\right\rceil=0$. Whence writing $p \equiv\left\lceil\bigwedge_{m} b_{m}\right\rceil$, we have $p \cdot \bigwedge_{n} a_{n} \equiv\left(\bigwedge_{n} a_{n}\right) \cdot p=$ 0 using Lemma 22. Observing that $\bigwedge_{n} b_{n} \leq p$ and using Lemma 21 we also have $p^{\perp} \cdot \bigwedge_{n} b_{n}=0$. We then calculate $p \cdot a=p \cdot\left(\bigwedge_{n} a_{n} \oslash a \wedge b\right)=p \cdot(a \wedge b)$ and similarly $p^{\perp} \cdot b=p^{\perp} \cdot(a \wedge b)$.

Returning to the problem of whether $\ell \leq a \wedge b$, we have

$$
\begin{aligned}
\ell=p \cdot \ell \otimes p^{\perp} & \ell \leq p \cdot a \otimes p^{\perp} \cdot b \\
& =p \cdot(a \wedge b) \emptyset p^{\perp} \cdot(a \wedge b)=a \wedge b .
\end{aligned}
$$

Whence $a \wedge b$ is the infimum of $a$ and $b$.

The presence of finite infima and suprema in $\omega$-complete effect monoids prevents certain subtleties with respect to the relation and existence of infima and suprema in an order interval:

Corollary 40. Let $a \leq b$ be elements of an $\omega$-complete effect monoid $M$, and let $S$ be a non-empty subset of $[a, b]_{M}$.

Then $S$ has a supremum (infimum) in $M$ if and only if $S$ has a supremum (infimum) in $[a, b]_{M}$. These these suprema (infima) coincide.

Proof. It is clear that if $S$ has a supremum in $M$, then this is also the supremum in $[a, b]_{M}$. For the converse, suppose that $S$ has a supremum $\bigvee S$ in $[a, b]_{M}$, and let $u$ be an upper bound for $S$ in $M$; in order to show that $\bigvee S$ is the supremum of $S$ in $M$ too, we must prove that $\bigvee S \leq u$. Note that $b \wedge u$ is an upper bound for $S$. Indeed, given $s \in S \subseteq[a, b]_{M}$ we have $s \leq b$, and $s \leq u$, so $s \leq b \wedge u$. Moreover, one easily sees that $b \wedge u \in[a, b]_{M}$ using the fact that $S$ is non-empty. Whence $b \wedge u$ is an upper bound for $S$ in $[a, b]_{M}$, and so $\bigvee S \leq b \wedge u \leq u$, making $\bigvee S$ the supremum of $S$ in $M$. Similar reasoning applies to infima of $S$.

Corollary 41. Given an element $a$ and a non-empty subset $S$ of an $\omega$-complete effect monoid $M$ such that $a \emptyset s$ exists for all $s \in S$,

1. the supremum $\bigvee S$ exists iff $\bigvee a \oslash S$ exists, and in that case $a \otimes \vee S=\bigvee a \otimes S$;

2. the infimum $\wedge S$ exists iff $\wedge a \emptyset S$ exists, and in that case $a \oslash \wedge S=\wedge a \emptyset S$.

Proof. The map $a \oslash():\left[0, a^{\perp}\right]_{M} \rightarrow[a, 1]_{M}$, being an order isomorphism, preserves and reflects suprema and infima. Now apply Corollary 40.

Now that we know more about the existence of suprema and infima, we set our sights on proving that multiplication interacts with suprema and infima as desired, namely that it preserves them. To do this we introduce a partial division operation. 
Definition 42. Given elements $a \leq b$ of an $\omega$-complete effect monoid, set $a / b \equiv \emptyset_{n=0}^{\infty} a \cdot\left(b^{\perp}\right)^{n}$. Note that the sum exists, because $\emptyset_{n=0}^{N} a \cdot\left(b^{\perp}\right)^{n} \leq \emptyset_{n=0}^{\infty} b \cdot\left(b^{\perp}\right)^{n} \equiv\lceil b\rceil$ for all $N$.

Lemma 43. Let $b$ be an element of an $\omega$-complete effect monoid $M$.

1. $b / b=\lceil b\rceil$.

2. $\left(a_{1} \oslash a_{2}\right) / b=a_{1} / b \otimes a_{2} / b$ for all summable $a_{1}, a_{2} \in M$ with $a_{1} \otimes a_{2} \leq b$.

3. $(a \cdot b) / b=a \cdot\lceil b\rceil$ for all $a \in M$.

4. $(a / b) \cdot b=a$ for all $a \in M$ with $a \leq b$.

5. $\{a \cdot b ; a \in M\} \equiv M b=[0, b]_{M} \equiv\{a ; a \in M ; a \leq b\}$.

6. The maps $a \mapsto a \cdot b, b \cdot a: M\lceil b\rceil \rightarrow M b$ are order isomorphisms.

Proof. Points 1 and 2 are easy, and left to the reader. Concerning 3 , first note that

$$
(a \cdot b) / b \equiv \bigotimes_{n=0}^{\infty} a \cdot b \cdot\left(b^{\perp}\right)^{n} \leq a \cdot \bigotimes_{n=0}^{\infty} b \cdot\left(b^{\perp}\right)^{n}=a \cdot\lceil b\rceil .
$$

Thus $(a \cdot b) / b \leq a \cdot\lceil b\rceil$. Since similarly $\left(a^{\perp} \cdot b\right) / b \leq a^{\perp} \cdot\lceil b\rceil$, we get, using 2 ,

$\lceil b\rceil=b / b=(a \cdot b) / b \otimes\left(a^{\perp} \cdot b\right) / b \leq a \cdot\lceil b\rceil \otimes a^{\perp} \cdot\lceil b\rceil=\lceil b\rceil$, forcing $(a \cdot b) / b=a \cdot\lceil b\rceil$ (see Lemma 24). For point 4, note that given $a, b \in M$ with $a \leq b$ we have $a=a \cdot\lceil b\rceil$ (by Lemma 21, since $a \leq b \leq\lceil b\rceil$,) and so

$$
\begin{array}{rlrl}
a=a \cdot\lceil b\rceil & =(a \cdot b) / b & & \text { by point } 3 \\
\equiv \bigotimes_{n=0}^{\infty} a \cdot b \cdot\left(b^{\perp}\right)^{n} & =\bigotimes_{n=0}^{\infty} a \cdot\left(b^{\perp}\right)^{n} \cdot b & & \text { by Lemma } 19 \\
& \leq\left(\bigotimes_{n=0}^{\infty} a \cdot\left(b^{\perp}\right)^{n}\right) \cdot b=(a / b) \cdot b .
\end{array}
$$

Since similarly $b \ominus a \leq((b \ominus a) / b) \cdot b$, we get

$$
\begin{aligned}
b=a \emptyset(b \ominus a) \leq(a / b) \cdot b \emptyset((b \ominus a) / b) \cdot b \\
=(b / b) \cdot b=\lceil b\rceil \cdot b=b,
\end{aligned}
$$

which forces $a=(a / b) \cdot b$. For point 5 , note that $M b, b M \subseteq$ $[0, b]_{M}$ since $b \cdot a, a \cdot b \leq b$ for all $a \in M$, and $[0, b]_{M} \subseteq M b$, because $a=(a / b) \cdot b$ for any $a \in[0, b]_{M}$ by point 4 .

Finally, concerning point 6: the maps $a \mapsto a \cdot b: M\lceil b\rceil \rightarrow$ $M b$ and $a \mapsto a / b: M b \rightarrow M\lceil b\rceil$ are clearly order preserving, and each other's inverse by points 3 and 4 , and thus order isomorphisms. The proof that $a \mapsto b \cdot a: M\lceil b\rceil \rightarrow M b$ is an order isomorphism follows along entirely similar lines, but involves $b \backslash a$ defined by $b \backslash a \equiv \emptyset_{n}\left(b^{\perp}\right)^{n} \cdot a$ and uses the fact that $b M=[0, b]_{M}=M b$.

Remark 44. From the previous lemma it follows that any $\omega$ complete effect monoid is a so called effect divisoid [55, §195].
The converse is false: later on we will show that any $\omega$ complete effect monoid is commutative, but there exists a non-commutative effect divisoid. [7, Ex. 4.3.9 $]^{2}$.

Finally, we can prove that multiplication is indeed normal:

Theorem 45. Let $b$ and $b^{\prime}$ be elements of an $\omega$-complete effect monoid $M$, and let $S \subseteq M$ be any (potentially uncountable or non-directed) non-empty subset.

1. If $\bigvee S$ exists, then so does $\bigvee_{s \in S} b \cdot s \cdot b^{\prime}$, and $b \cdot(\bigvee S) \cdot b^{\prime}=$ $\bigvee_{s \in S} b \cdot s \cdot b^{\prime}$.

2. If $\bigwedge S$ exists, then so does $\bigwedge_{s \in S} b \cdot s \cdot b^{\prime}$, and $b \cdot(\bigwedge S) \cdot b^{\prime}=$ $\bigwedge_{s \in S} b \cdot s \cdot b^{\prime}$.

Proof. Suppose that $\bigvee S$ exists. We will prove that $b \cdot \bigvee S=$ $\bigvee_{s \in S} b \cdot s$, and leave the remainder to the reader. Note that $b \cdot():[0,\lceil b\rceil]_{M} \rightarrow[0, b]_{M}$, being an order isomorphism by Lemma 43(6), preserves suprema and infima. The set $S$ need, however, not be part of $[0,\lceil b\rceil]_{M}$, so we consider instead of $b$ the element $b^{\prime} \equiv b \otimes\lceil b\rceil^{\perp}$, for which $\left\lceil b^{\prime}\right\rceil=$ $\lceil b\rceil \vee\lceil b\rceil^{\perp}=1$ by Lemma 38. We then get an order isomorphism $b^{\prime} \cdot(): M \rightarrow\left[0, b^{\prime}\right]_{M}$, which preserves suprema, so that $b^{\prime} \cdot \bigvee S$ is the supremum of $b^{\prime} \cdot S$ in $\left[0, b^{\prime}\right]_{M}$, and hence in $M$, by Corollary 40 . Then

$$
\begin{aligned}
\left(b \otimes\lceil b\rceil^{\perp}\right) \cdot \bigvee S & =\bigvee_{s \in S}\left(b \otimes\lceil b\rceil^{\perp}\right) \cdot s \\
& \leq \bigvee_{s \in S} b \cdot s \emptyset \bigvee_{s^{\prime} \in S}\lceil b\rceil^{\perp} \cdot s^{\prime} \\
\leq b \cdot \bigvee S \otimes\lceil b\rceil^{\perp} \cdot \bigvee S & =\left(b \otimes\lceil b\rceil^{\perp}\right) \cdot \bigvee S \\
\text { forces } \bigvee_{s \in S} b \cdot s=b \cdot \bigvee S & (\text { see Lemma 24). }
\end{aligned}
$$

\section{Boolean algebras, halves and convexity}

We are ready to study the two important types of idempotents in an effect monoid: those that are Boolean and those that are halvable.

Definition 46. We say an element $a$ of an effect monoid $M$ is Boolean when each $b \leq a$ is idempotent. We say an effect monoid is Boolean when 1 is Boolean.

Proposition 47. The set of idempotents $P(M)$ of an effect monoid $M$ is a Boolean algebra. Thus an effect monoid is Boolean iff it is a Boolean algebra.

Proof. First we will show that in fact $p \cdot q=p \wedge q$ for $p, q \in$ $P(M)$, where the infimum $\wedge$ is taken in $M$. Using Lemma 22, we see $(p \cdot q)^{2}=p \cdot q \cdot p \cdot q=p \cdot p \cdot q \cdot q=p \cdot q$ and so $p \cdot q$ is an idempotent. Let $r \leq p, q$. Then $r \cdot p=r$ and $r \cdot q=r$ so that $r \cdot p \cdot q=r$, and hence $r \leq p \cdot q$ by Lemma 21, which shows $p \cdot q=p \wedge q$. As the complement is an order antiisomorphism, we find $p \vee q=\left(p^{\perp} \wedge q^{\perp}\right)^{\perp}$ and hence $P(M)$ is a complemented lattice. It remains to show that it satisfies distributivity: $p \wedge(q \vee r)=(p \wedge q) \vee(p \wedge r)$. By uniqueness of complements, it is easily shown that $p \vee q=p \otimes\left(p^{\perp} \cdot q\right)=$ $q \otimes\left(p \cdot q^{\perp}\right)$. The remainder is a straightforward exercise in

${ }^{2}$ Cho shows that there is a non-commutative division effect monoid. Any division effect monoid is an effect divisoid as well. 
writing out the expressions $p \wedge(q \vee r)$ and $(p \wedge q) \vee(p \wedge r)$ and noting that they are equal.

Proposition 48. If $M$ is $\omega$-complete, then the Boolean algebra of projections $P(M)$ is $\omega$-complete.

Proof. Let $A \subseteq P(M)$ be a countable subset. Pick an enumeration of its elements $p_{1}, p_{2}, \ldots$. Let $q_{n}$ be iteratively defined as $q_{1} \equiv p_{1}$ and $q_{n} \equiv q_{n-1} \vee p_{n}$. Then the $q_{n}$ form an increasing sequence and hence it has a supremum $q$. We claim that $q$ is also the supremum of $A$. Of course $q \geq q_{n} \geq p_{n}$ and hence $q$ is an upper bound. Suppose that $r \geq p_{n}$ for all $n$. Then $r \geq q_{1}$, and hence by induction if $r \geq q_{n}$ then $r \geq q_{n} \vee p_{n}=q_{n+1}$. Hence also $r \geq q$.

Proposition 49. Let $M$ be an $\omega$-complete Boolean effect monoid. Then $M$ is an $\omega$-complete Boolean algebra.

Proof. By Propositions 47 and $48 P(M)$ is an $\omega$-complete Boolean algebra. But by assumption every element of $M$ is an idempotent, and hence $M=P(M)$.

The counterpart to the Boolean effect monoids, are the halvable effect monoids

Definition 50. We say that an element $a$ of an effect algebra $E$ is halvable when $a=b \otimes b$ for some $b \in E$. We say an effect algebra is halvable when 1 is halvable.

A halvable $\omega$-complete effect monoid actually has much more structure then might be apparent:

Definition 51. Let $E$ be an effect algebra. We say $E$ is convex if there exists an action $\cdot[0,1] \times E \rightarrow E$, where $[0,1]$ is the standard real unit interval, satisfying the following axioms for all $a, b \in E$ and $\lambda, \mu \in[0,1]$ :

- $\lambda \cdot(\mu \cdot a)=(\lambda \mu) \cdot a$.

- If $\lambda+\mu \leq 1$, then $\lambda \cdot a \perp \mu \cdot a$ and $\lambda \cdot a \emptyset \mu \cdot a=(\lambda+\mu) \cdot a$.

- If $a \perp b$, then $\lambda \cdot a \perp \lambda \cdot b$ and $\lambda \cdot(a \emptyset b)=\lambda \cdot a \emptyset \lambda \cdot b$.

- $1 \cdot a=a$.

In a convex effect monoid we will usually write the convex action without any symbol in order to distinguish it from the multiplication coming from the monoid structure. So if $\lambda \in[0,1]$ is a real number and $a, b \in M$ is a convex effect monoid, then we write $\lambda(a \cdot b)$. Note that a priori it is not clear whether $\lambda(a \cdot b)=(\lambda a) \cdot b=a \cdot(\lambda b)$.

Proposition 52. Let $M$ be a halvable $\omega$-complete effect monoid. Then $M$ is convex.

Proof. Pick any $a \in M$ with $a \emptyset a=1$. Let $q=\frac{m}{2^{n}}$ be a dyadic rational number with $0 \leq m \leq 2^{n}$. We define a corresponding element $\bar{q} \in M$ by $\bar{q}=m a^{n}$, which is easily seen to be independent of the choice of $m$ and $n$. This yield an action $(q, s) \mapsto \bar{q} \cdot s$ that satisfies all axioms of Definition 51 restricted to dyadic rationals.

Assume $\lambda \in(0,1]$. Let $0 \leq q_{1}<q_{2}<\ldots$ be a strictly increasing sequence of dyadic rationals with $\sup q_{i}=\lambda$. We will define $\bar{\lambda} \in M$ by $\bigvee_{i} \overline{q_{i}}$, but first we have to show that it is independent of the choice of the sequence and that it coincides with the definition just given for dyadic rationals. So assume $0 \leq p_{1}<p_{2}<\ldots$ is any other sequence of dyadic rationals with $\sup p_{i}=\lambda$. For any $p_{i}$ we can find a $q_{j}$ with $p_{i} \leq q_{j}$, so $\overline{p_{i}} \leq \overline{q_{j}}$, hence $\bigvee_{i} \overline{p_{i}} \leq \bigvee_{j} \overline{q_{j}}$. As the situation is symmetric between the sequences, we also have $\bigvee_{j} \overline{q_{j}} \leq \bigvee_{i} \overline{p_{i}}$ and so $\bigvee_{j} \overline{q_{j}}=\bigvee_{i} \overline{p_{i}}$. Hence $\bar{\lambda}$ is independent of the choice of sequence. Next, assume $\lambda \equiv q$ is a non-zero dyadic rational. Pick $m$ with $2^{-m} \leq q$. Then $q_{n} \equiv$ $q-2^{-(m+n)}$ is a sequence of dyadic rationals with $\sup q_{n}=q$. We have $\bigvee_{n} \overline{q-2^{-(m+n)}}=\bar{q} \ominus \bigwedge_{n} \overline{2^{-(m+n)}}=\bar{q} \ominus \bigwedge_{n} a^{m+n}=\bar{q}$, (where in the last step we used Lemma 28) so both definitions of $\bar{q}$ coincide. As a result we are indeed justified to define $\bar{\lambda}=\bigvee_{i} \overline{q_{i}}$. We can then define an action by $(\lambda, s) \mapsto \bar{\lambda} \cdot s$. As both addition and multiplication preserve suprema by Theorem 45, it is straightforward to show that this action indeed satisfies all the axioms of a convex action.

\section{Embedding theorems}

We now have what we need to show that any $\omega$-complete effect monoid embeds into a direct sum of a Boolean effect monoid and a halvable one, which lies at the heart of our results.

Lemma 53. The ceiling $\lceil a\rceil$ of a halvable element $a$ of an $\omega$-complete effect monoid is halvable too.

Proof. Write $a \equiv b \otimes b$. We compute

$$
\begin{aligned}
\lceil a\rceil \equiv \emptyset_{n} a \cdot\left(a^{\perp}\right)^{n} & =\emptyset_{n}(b \otimes b) \cdot\left(a^{\perp}\right)^{n} \\
= & \left(\emptyset_{n} b \cdot\left(a^{\perp}\right)^{n}\right) \otimes\left(\emptyset_{n} b \cdot\left(a^{\perp}\right)^{n}\right),
\end{aligned}
$$

and hence it is indeed halvable.

Proposition 54. Each $\omega$-complete effect monoid $M$ has a subset $E \subseteq M$

1. that is a maximal collection of non-zero orthogonal idempotents, and

2. such that each element of $E$ is either halvable or Boolean.

Proof. Let $H$ be a maximal collection of non-zero orthogonal halvable idempotents of $M$, and let $E$ be a maximal collection of non-zero orthogonal idempotents of $M$ that extends $H$. (Such sets $E$ and $H$ exist by Zorn's Lemma). By definition, $E$ is a maximal collection of non-zero orthogonal idempotents of $M$, so the only thing to prove is that each $e \in E \backslash H$ is Boolean. Hence, let $a$ be an element of $M$ below some $e \in$ $E \backslash H$; we must show that $a$ is an idempotent. Note that 2( $a^{\perp}$. $a) \equiv a^{\perp} \cdot a \emptyset a^{\perp} \cdot a$ (which exists by e.g. Lemma 27) is halvable, and $2\left(a^{\perp} \cdot a\right) \leq e$, because $2\left(a^{\perp} \cdot a\right) \cdot e=2\left(a^{\perp} \cdot a \cdot e\right)=2\left(a^{\perp} \cdot a\right)$. Then the idempotent $\left\lceil 2 a \cdot a^{\perp}\right\rceil \leq e$, which is halvable by Lemma 53, is orthogonal to all $h \in H$ (since it is below $e$ ) and must therefore be zero lest it contradict the maximality of $H$. In particular, $a^{\perp} \cdot a=0$ since $a^{\perp} \cdot a \leq\left\lceil 2 a^{\perp} \cdot a\right\rceil=0$, and so $a$ is an idempotent by Lemma 20. Whence $e$ is Boolean. 
Note that the only idempotent that is both Boolean and halvable is zero, and hence each element in the above set is either Boolean or halvable.

Proposition 55. Given a maximal orthogonal collection of non-zero idempotents $E$ of an $\omega$-complete effect monoid $M$, the map $a \mapsto(a \cdot e)_{e}: M \longrightarrow \bigoplus_{e \in E} M e$ is an embedding of effect monoids.

Proof. The map obviously maps 1 to 1 , and preserves addition. Hence it also preserves the complement and the order. By Lemma 22 we have $(a \cdot e) \cdot(b \cdot e)=(a \cdot b) \cdot(e \cdot e)=(a \cdot b) \cdot e$, and so the map also preserves the multiplication. It remains to show that the map is order reflecting. Note that if we had $\varnothing E=1$, then for any $a$ by Theorem 45 , we have $a=$ $a \cdot 1=a \cdot \emptyset_{e \in E} e=\emptyset_{e \in E} a \cdot e$, and hence if $a \cdot e \leq b \cdot e$ for all $e \in E$ we have $a=\emptyset_{e \in E} a \cdot e \leq \emptyset_{e \in E} b \cdot e=b$, which proves that it is indeed order reflecting.

So let us prove that $\emptyset E=1$. Suppose $u$ is an upper bound for $E$; we must show that $u=1$. Note that $\lfloor u\rfloor$ is an upper bound for $E$ too, since $E$ contains only idempotents. It follows that the idempotent $\lfloor u\rfloor^{\perp}=\left\lceil u^{\perp}\right\rceil$ is orthogonal to all $e \in E$, which is impossible (by maximality of $E$ ) unless $\left\lceil u^{\perp}\right\rceil=0$. Hence $\left\lceil u^{\perp}\right\rceil=0$, and thus $u^{\perp}=0$.

Theorem 56. Let $M$ be an $\omega$-complete effect monoid. Then there exist $\omega$-complete effect monoids $M_{1}$ and $M_{2}$ where $M_{1}$ is convex, and $M_{2}$ is an $\omega$-complete Boolean algebra such that $M$ embeds into $M_{1} \oplus M_{2}$.

Proof. Let $E=H \cup B$ be a maximal collection of non-zero orthogonal idempotents of Proposition 54 such that the idempotents $p \in H$ are halvable, while the $q \in B$ are Boolean.

Let $M_{1} \equiv \bigoplus_{p \in H} p M$ and $M_{2} \equiv \bigoplus_{q \in B} q M$. It is easy to see that $M_{1}$ is then again halvable and $M_{2}$ is Boolean. By Propositions 52 and 49 we get that $M_{1}$ is convex while $M_{2}$ is an $\omega$-complete Boolean algebra. By the previous proposition $M$ embeds into $\bigoplus_{p \in E} p M \cong M_{1} \oplus M_{2}$.

One might be tempted to think that the above result could be strengthened to an isomorphism. The following example shows that this is not the case:

Example 57. Let $X_{1}$ and $X_{2}$ be uncountably infinite sets, and let $A$ be the set of all pairs of functions

$$
A \equiv\left\{\left(f_{1}: X_{1} \rightarrow[0,1], f_{2}: X_{2} \rightarrow\{0,1\}\right)\right\} .
$$

Let $S_{0}, S_{1} \subseteq A$ be subsets where both functions are unequal to 0 respectively 1 only at a countable number of spots:

$$
\begin{aligned}
& S_{0} \equiv\left\{\left(f_{1}, f_{2}\right) ; \text { both }\left[\begin{array}{l}
\left\{x_{1} \in X_{1} ; f_{1}\left(x_{1}\right) \neq 0\right\} \\
\left\{x_{2} \in X_{2} ; f_{2}\left(x_{2}\right) \neq 0\right\}
\end{array} \text { countable }\right\}\right. \\
& S_{1} \equiv\left\{\left(f_{1}, f_{2}\right) ; \text { both }\left[\begin{array}{l}
\left\{x_{1} \in X_{1} ; f_{1}\left(x_{1}\right) \neq 1\right\} \\
\left\{x_{2} \in X_{2} ; f_{2}\left(x_{2}\right) \neq 1\right\}
\end{array} \text { countable }\right\}\right.
\end{aligned}
$$

Finally, define $M=S_{0} \cup S_{1}$. It is then straightforward to check that $M$ is a $\omega$-complete effect monoid. It is easy to see that $M$ has no maximal halvable idempotent, and hence for any $M_{1}$ halvable and $M_{2}$ Boolean, necessarily $M \neq M_{1} \oplus M_{2}$.

Though the embedding is not an isomorphism for $\omega$-complete effect monoids, when we assume full directed-completeness, we can derive a stronger result.

Lemma 58. Let $M$ be a directed-complete effect monoid. Then there is a maximal element that is summable with itself. That is: there is an $a \in M$ such that $a \perp a$ and for any other $b \geq a$ such that $b \perp b$, we have $b=a$. Furthermore,

1. $a \otimes a$ is an idempotent and

2. if $s \leq(a \oslash a)^{\perp}$, then $s$ is idempotent for any $s \in M$.

Proof. Write $A \equiv\{s \in M ; s \perp s\}$. We will show that $A$ has a maximal element using Zorn's Lemma. To this end, suppose $D \subseteq A$ is a chain. We have to show that it has an upper bound in $A$. If $D$ is empty, then $0 \in A$ is clearly an upper bound, so we may assume that $D$ is not empty. Define $s \equiv \bigvee D$. It is sufficient to show $s \perp s$ as then $s \in A$.

Assume $d, d^{\prime} \in D$. We claim $d \perp d^{\prime}$. Indeed, assuming without loss of generality that $d^{\prime} \leq d$, we see $d^{\prime} \leq d \leq d^{\perp}$ and so $d \perp d^{\prime}$.

So $\bigvee_{d^{\prime} \in D} d \otimes d^{\prime}$ exists. As addition preserves suprema, we have $\bigvee_{d^{\prime} \in D} d \otimes d^{\prime}=d \emptyset \bigvee_{d^{\prime} \in D} d^{\prime}=d \otimes s$. Hence $\bigvee_{d \in D} d \emptyset s$ exists and $\bigvee_{d \in D} d \emptyset s=\left(\bigvee_{d \in D} d\right) \emptyset s=s \emptyset s$, so indeed $s \in A$. By Zorn's Lemma we know there is a maximal element of $A$. Pick such a maximal element $a \in A$.

Next we will show that $a \oslash a$ is idempotent. Define $b \equiv$ $(a \otimes a)^{\perp}$. Note that $b \cdot b^{\perp}$ is summable with itself. As a result $b=b \cdot 1 \geq b \cdot\left(b \cdot b^{\perp} \emptyset b \cdot b^{\perp}\right)=b^{2} \cdot b^{\perp} \oslash b^{2} \cdot b^{\perp}$. Now note that

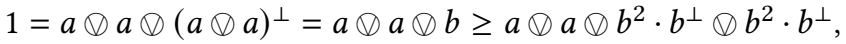
and hence $a \otimes b^{2} \cdot b^{\perp}$ is summable with itself. Since $a$ is maximal with this property we must have $b^{2} \cdot b^{\perp}=0$. But then $\left(b \cdot b^{\perp}\right)^{2}=b^{2} \cdot b^{\perp} b^{\perp}=0$ and so $b \cdot b^{\perp}=0$ by Lemma 28 . Hence $b^{\perp}=a \otimes a$ is indeed idempotent.

Now let $s \leq(a \otimes a)^{\perp} \equiv b$. We have to show that $s$ is idempotent. By Lemma $27 s \cdot s^{\perp}$ is summable with itself and so $s \cdot s^{\perp} \otimes s \cdot s^{\perp} \leq b \equiv(a \bigotimes a)^{\perp}$ by Lemma 25. Thus $a \emptyset a \perp$ $s \cdot s^{\perp} \oslash s \cdot s^{\perp}$ and so $a \emptyset s \cdot s^{\perp}$ is summable with itself. By maximality of $a$ we must have $s \cdot s^{\perp}=0$, which shows that $s$ is indeed an idempotent.

Theorem 59. Given a directed-complete effect monoid $M$, there exists a convex directed-complete effect monoid $M_{1}$, and a complete Boolean algebra $M_{2}$ such that $M \cong M_{1} \oplus M_{2}$.

Proof. Let $a$ be a maximal element that is summable to itself from Lemma 58. It was shown that $p=(a \emptyset a)^{\perp}$ is an idempotent such that any $s \leq p$ is also an idempotent. Hence by an adaptation of Proposition 49 to directed-complete effect monoids, $p M$ is a complete Boolean algebra.

But of course $p^{\perp} M$ is halvable and hence by Proposition 52 $p^{\perp} M$ is convex. Hence letting $M=p^{\perp} M \oplus p M$ gives the desired result. 


\section{Order unit spaces}

In this section we will see that a convex $\omega$-complete effect monoid $M$ is isomorphic to the set of continuous functions of some basically disconnected compact Hausdorff space $X$ to the real unit interval, or equivalently by Gel'fand duality, isomorphic to the unit interval of an $\omega$-complete commutative $\mathrm{C}^{*}$-algebra. We do this by using a known representation of convex effect algebras as unit intervals of order unit spaces, and then apply Yosida's representation theorem for latticeordered vector spaces.

Definition 60. An order unit space (OUS) is an ordered vector space together with distinguished element $1 \in V$, such that for every $v \in V$, there is a $n \in \mathbb{N}$ such that $-n \cdot 1 \leq v \leq$ $n \cdot 1$. An OUS is called Archimedean provided that $v \leq \frac{1}{n} \cdot 1$ for all $n \in \mathbb{N}$, implies $v \leq 0$.

Definition 61. Given an OUS $V$, its unit interval $[0,1]_{V} \equiv$ $\{v \in V ; 0 \leq v \leq 1\}$ is a convex effect algebra with $a \perp b$ iff $a+b \leq 1$ and then $a \emptyset b=a+b$; $a^{\perp}=1-a$; and the convex structure being given by the obvious scalar multiplication. We say $V$ is $\omega$-complete/directed complete whenever $[0,1]_{V}$ is.

Lemma 62. Let $V$ be an order unit space. It is directed complete if and only if it is bounded directed complete; that is if every directed subset of $V$ with upper bound has a supremum. Similarly $V$ is $\omega$-complete iff it is bounded $\omega$ complete.

Proof. Since any subset of the unit interval is obviously bounded, any bounded $\omega$-complete/directed-complete order unit space is $\omega$-complete/directed complete. For the other direction, assume $S \subseteq V$ is some (countable) directed subset with upper bound $b \in V$. Pick any $a \in S$ and define $S^{\prime} \equiv$ $\{v ; v \in S ; a \leq v\}$. It is sufficient to show that $S^{\prime}$ has a supremum. Pick any $n \in \mathbb{N}, n \neq 0$ with $-n \cdot 1 \leq a, b \leq n \cdot 1$. Then clearly $\left\{\frac{1}{n}(s-a) ; s \in S^{\prime}\right\} \subseteq[0,1]_{V}$ has a supremum and so does $S^{\prime}$ as $v \mapsto n v+a$ is an order isomorphism.

Lemma 63. A bounded $\omega$-complete OUS is Archimedean.

Proof. Assume $V$ is a bounded $\omega$-complete OUS. Let $v \in V$ be given with $v \leq \frac{1}{n} 1$ for all $n \in \mathbb{N}$. As $a \mapsto-a$ is an order anti-isomorphism, all bounded directed subsets of $V$ have an infimum too, so $v \leq \bigwedge_{n} \frac{1}{n} 1=\left(\inf _{n} \frac{1}{n}\right) 1=0$ as desired.

Recall that the unit interval of an OUS is a convex effect algebra. In fact, every convex effect algebra is of this form.

Theorem 64. Let $M$ be a convex effect algebra. Then there exists an order unit space $V$ such that $M \cong[0,1]_{V}[22]$.

Proposition 65. Let $M$ be an $\omega$-complete convex effect monoid. Then the multiplication is "bilinear": $\lambda(a \cdot b)=$ $(\lambda a) \cdot b=a \cdot(\lambda b)$ for any $a, b \in M$ and $\lambda \in[0,1]$.

Proof. Clearly $n\left(a \cdot\left(\frac{1}{n} b\right)\right)=a \cdot b=n \frac{1}{n}(a \cdot b)$ and so $a \cdot\left(\frac{1}{n} b\right)=$ $\frac{1}{n}(a \cdot b)$. Hence $m\left(a \cdot\left(\frac{1}{n} b\right)\right)=a \cdot\left(\frac{m}{n} b\right)=\frac{m}{n}(a \cdot b)$ for any $m \leq n$.
We have shown the second equality for rational $\lambda$. With a similar argument one shows the first equality holds as well for rational $\lambda$. To prove the general case, let $a, b \in M$ and $\lambda \in[0,1]$ be given. Pick a sequence $0 \leq q_{1}<q_{2}<\ldots$ of rationals with $\sup _{n} q_{n}=\lambda$. Let $V$ be an OUS with $M \cong[0,1]_{V}$ (as a convex effect algebra), which exists due to Theorem 64. Note that the multiplication of $M$ is only defined on $[0,1]_{V}$. For $a \in[0,1]_{V}$ we have $a \leq\|a\| 1$ and so $\|a \cdot b\| \leq\|a\|\|b\|$ for any $b \in[0,1]_{V}$. Hence

$$
\begin{aligned}
& \|\lambda(a \cdot b)-a \cdot(\lambda b)\| \\
& \quad=\left\|\left(\lambda-q_{i}\right)(a \cdot b)+a \cdot\left(q_{i} b\right)-a \cdot(\lambda b)\right\| \\
& \quad=\left\|\left(\lambda-q_{i}\right)(a \cdot b)-a \cdot\left(\left(\lambda-q_{i}\right) b\right)\right\| \\
& \quad \leq\left(\lambda-q_{i}\right)\|a \cdot b\|+\left(\lambda-q_{i}\right)\|a\|\|b\| .
\end{aligned}
$$

The right-hand side vanishes as $i \rightarrow \infty$. Thus $\| \lambda(a \cdot b)-$ $a \cdot(\lambda b)=0 \|$. Since $V$ is Archimedean by Lemma 63, $\|\cdot\|$ is a proper norm so that then $\lambda(a \cdot b)-a \cdot(\lambda b)=0$. That $\lambda(a \cdot b)=(\lambda a) \cdot b$ follows similarly.

Theorem 66 (Yosida [57], cf. [54]). Let $V$ be a norm-complete lattice-ordered Archimedean OUS. Denote by $\Phi$ the Yosida spectrum of $V$ : the compact Hausdorff space of real-valued finite-suprema-preserving unital linear functionals on $V$ with the induced pointwise topology of $\mathbb{R}^{V}$. Then the map $\vartheta: V \rightarrow C(\Phi)$ given by $\vartheta(v)(\varphi)=\varphi(v)$ is an order isomorphism.

Remark 67. In fact a stronger result is true: the category of compact Hausdorff spaces with continuous maps between them is dually equivalent to the category of lattice-ordered norm-complete Archimedean order unit spaces with linear unital finite-supremum-preserving maps between them. [54]

Theorem 68. Let $M$ be a convex $\omega$-complete effect monoid. Then $M \cong[0,1]_{C(X)}$ for some basically disconnected Hausdorff space $X$. If $M$ is even directed complete, then $X$ is extremally disconnected.

Proof. The effect monoid $M$ is a lattice by Theorem 39. By Theorem 64 there is an OUS $V$ such that $M \cong[0,1]_{V}$ as a convex effect algebra. It is easy to see that $V$ is a lattice as well as any supremum reduces to one in the interval $[0,1]_{V}$. Note $V$ is bounded $\omega$-complete by Lemma 62 and hence Archimedean by Lemma 63, and norm complete by [56, Lemma 1.2]. Then by Theorem 66 we have $V \cong$ $C(\Phi)$, where $\Phi$ is the Yosida spectrum of $V$. Recall $C(\Phi)$ is bounded $\omega$-complete iff $\Phi$ is basically disconnected and $C(\Phi)$ is bounded directed complete iff $\Phi$ is extremally disconnected [15, 1H, 3N.5 \& 3N.6]. The multiplication of $M$ induces a multiplication on $[0,1]_{C(\Phi)}$, which we will denote by $f * g$ to distinguish it from the pointwise multiplication $f \cdot g$. To prove the Theorem, it remains to be shown that $f * g=f \cdot g$.

Pick any $\varphi \in \Phi$. It is sufficient to show that $\varphi(f * g)=$ $\varphi(f) \varphi(g)$ - indeed then $(f * g)(\varphi)=\varphi(f * g)=\varphi(f) \varphi(g)=$ $f(\varphi) g(\varphi)=(f \cdot g)(\varphi)$. The remainder of the proof is based 
on [49, Lemma 5.26]. Write $d(a, b) \equiv a \vee b-a \wedge b$ for either $a, b \in[0,1]$ or $a, b \in[0,1]_{C(\Phi)}$. Note $f *(g \vee h) \leq$ $(f * g) \vee(f * h)$ and $f *(g \wedge h) \geq(f * g) \wedge(f * h)$, hence $d(f * g, f * h) \leq f * d(g, h)$ for $f, g, h \in[0,1]_{C(\Phi)}$. So in particular $d(f * g, f *(\varphi(g) 1)) \leq f * d(g, \varphi(g) 1) \leq$ $d(g, \varphi(g) 1)$. The multiplication $*$ is "bilinear" by Proposition 65 , so $f *(\varphi(g) 1)=\varphi(g) f$. By definition $\varphi$ preserves infima, suprema and 1 and so $d(\varphi(f), \varphi(g))=\varphi(d(f, g))$ for any $f, g \in[0,1]_{C(\Phi)}$. Combining the last three facts, we see

$$
\begin{aligned}
d(\varphi(f * g), \varphi(f) \varphi(g)) & =\varphi(d(f * g, f *(\varphi(g) 1))) \\
\leq \varphi(d(g, \varphi(g) 1)) & =d(\varphi(g), \varphi(g))=0 .
\end{aligned}
$$

So indeed $\varphi(f * g)=\varphi(f) \varphi(g)$, hence $f * g=f \cdot g$.

Remark 69. The previous theorem can also be proven using Kadison's representation Theorem [36], which states that any norm-complete Archimedean OUS with a bilinear and positive product is isomorphic to $C(X)$. This requires one to show that the product on $[0,1]_{V}$ extends to a bilinear and positive product on the entirety of $V$, which can be done, but is a bit tedious (cf. [53, Theorem 46]).

\section{Main theorems}

We now collect our previous results and prove our main theorems.

Theorem 70. Let $M$ be an $\omega$-complete effect monoid. Then there exists a basically disconnected compact Hausdorff space $X$, and an $\omega$-complete Boolean algebra $B$ such that $M$ embeds into $[0,1]_{C(X)} \oplus B$.

Proof. By Theorem 56 there exist $\omega$-complete effect monoids $M_{1}$ and $M_{2}$ such that $M$ embeds into $M_{1} \oplus M_{2}$, where $M_{1}$ is convex and $M_{2}$ is an $\omega$-complete Boolean algebra. By Theorem $68 M_{1}=[0,1]_{C(X)}$ for a basically disconnected compact Hausdorff space $X$.

Theorem 71. Let $M$ be a directed-complete effect monoid. Then there exists an extremally-disconnected compact Hausdorff space $X$, and an complete Boolean algebra $B$ such that $M \cong[0,1]_{C(X)} \oplus B$.

Proof. Same as previous theorem but using Theorem 59.

Corollary 72. Let $M$ be an $\omega$-complete effect monoid. Then $M$ is commutative.

Proof. $M$ embeds into $[0,1]_{C(X)} \oplus B$ where $B$ is a Boolean algebra and $X$ is a basically disconnected compact Hausdorff space. The multiplication in $B$ is given by the join and hence is commutative, while the multiplication in $C(X)$ is given by pointwise multiplication in the real numbers and hence is also commutative. The multiplication of $M$ is then necessarily also commutative.

Theorem 73. Let $M$ be an $\omega$-complete effect monoid with no non-trivial zero divisors. Then either $M=\{0\}, M=\{0,1\}$ or $M \cong[0,1]$.
Proof. Assume that $M \neq\{0,1\}$ and $M \neq\{0\}$. We remark first that for any idempotent $p \in M$ we have $p \cdot p^{\perp}=0$, and hence by the lack of non-trivial zero divisors we must have $p=0$ or $p=1$. Since $M \neq\{0,1\}$, there is an $s \in M$ such that $s \neq 0,1$, and hence we must have $s \cdot s^{\perp} \neq 0$. By Lemma 27 we then have an element $2\left(s \cdot s^{\perp}\right)$ that is halvable. Hence by Lemma $53\left\lceil 2 s \cdot s^{\perp}\right\rceil$ is also halvable. As this ceiling is an idempotent it must be equal to 1 or to 0 . If it were zero then $2 s \cdot s^{\perp} \leq\left\lceil 2 s \cdot s^{\perp}\right\rceil=0$, which contradicts $s \cdot s^{\perp} \neq 0$. So $1=\left\lceil 2 s \cdot s^{\perp}\right\rceil$ is halvable. By Proposition $52, M$ is then convex. Hence, by Theorem $68 M=[0,1]_{C(X)}$ for some basically disconnected $X$. We will show that $X$ has a single element, which will complete the proof.

As idempotents of $[0,1]_{C(X)}$ correspond to clopens of $X$, there are only two clopens in $X$, namely $X$ and $\emptyset$. Reasoning towards contradiction, assume there are $x, y \in X$ with $x \neq y$. By Urysohn's lemma we can find $f \in C(X)$ with $0 \leq f \leq 1, f(x)=0$ and $f(y)=1$. $U_{x} \equiv f^{-1}\left(\left[0, \frac{1}{3}\right)\right.$ and $U_{y} \equiv f^{-1}\left(\left(\frac{2}{3}, 1\right]\right)$ are two open sets with disjoint closure. Using Urysohn's lemma again, we can find $g \in C(X)$ with $g\left(\overline{U_{x}}\right)=\{0\}$ and $g\left(\overline{U_{y}}\right)=\{1\}$. As $X$ is basically disconnected, we know $\overline{\operatorname{supp} g}$ is clopen. We cannot have $\overline{\operatorname{supp} g}=\emptyset$ as $y \in U_{y} \subseteq \overline{\operatorname{supp} g}$. Hence $\overline{\operatorname{supp} g}=X$. But then $x \in U_{x} \subseteq$ $X \backslash \overline{\operatorname{supp} g}=\emptyset$. Contradiction. Apparently $X$ has only one point and so $M \cong[0,1]$.

Remark 74. In [7] it is shown that any $\sigma$-effectus satisfying a natural property called normalisation (basically, that any substate can be normalised to a state) must have scalars without zero divisors. We have hence also completely classified the allowable scalars in a $\sigma$-effectus satisfying normalisation. The details are worked out in the follow-up work [9].

Remark 75. As noted in Example 9, the unit interval of any partially ordered ring where the sum and product of positive elements is again positive forms an effect monoid. Hence the previous results can also be seen as, to our knowledge, new results for $\sigma$-Dedekind-complete ordered rings. Although it is not clear how our results for the unit interval can be extended to results concerning the entire ring, our results might eventually lead to a version of the characterisation of the real numbers as the unique Dedekind-complete ordered field, where the condition of being a field can be weakened to being a domain (i.e. a ring without non-trivial zero-divisors).

\section{Conclusion and outlook}

We have shown that any $\omega$-complete effect monoid embeds into a direct sum of a Boolean algebra and the set of continuous functions from a given Hausdorff space $X$ into the real unit interval $[0,1]$. As a result, the only $\omega$-complete effect monoids without any zero divisors are either degenerate $(\{0\})$, the Booleans $(\{0,1\})$, or the real unit interval $([0,1])$, resulting in a dichotomy of sharp logic and fuzzy probabilistic logic. 
The structure of an $\omega$-complete effect monoid; partial addition, involution, multiplication and directed limits; captures in a sense the structure present in the real unit interval and hence these results give a foundational underpinning to why the unit interval should be the designated structure of the scalars in any non-sharp physical or logical theory.

An interesting follow-up question is to consider whether the assumption of $\omega$-completeness can be weakened somehow. There exist pathological (e.g. non-commutative) effect monoids ([7, Ex. 4.3.9] and [53, Cor. 51]), but all the known ones are 'non-Archimedean' in some way, having infinitesimals for example. Once a satisfactory definition for an Archimedean effect monoid has been established, it might be possible to show that it embeds into a directedcomplete effect monoids, similar to the Dedekind-MacNeille completion of a poset into a complete lattice. If this is the case, then we can essentially get rid of the assumption of $\omega$-completeness.

Another matter that deserves further investigation is the categorical aspect of our results. It can be shown that Theorem 71 has the following consequence: the category of directed-complete effect monoids with effect monoid homomorphisms between them (which need not preserve suprema) is dually equivalent to the category which has as objects pairs $(X, S)$, where $S \subseteq X$ is a clopen subset of a extremally disconnected compact Hausdorff space $X$, and where morphisms $(X, S) \rightarrow(Y, T)$ are continuous maps $f: X \rightarrow Y$ satisfying $f(S) \subseteq T$. Obtaining a similar duality for $\omega$-complete effect monoids would in our estimation be rather non-trivial, but as it involves $\omega$-complete algebras, it can perhaps use results analogous to those of Ref. [14].

In a forthcoming article [9], we apply these results to study $\sigma$-effectuses [7]. Continuing that line of research, it seems possible to make a "reconstruction of quantum theory" $[6,26]$ that does not a priori refer to the structure of the real unit interval, ${ }^{3}$ for instance by adopting the effectusbased axioms of Ref. [47].

Finally, when the associative bilinear multiplication of an effect monoid is replaced by a binary operation satisfying axioms related to the Lüders product $(a, b) \mapsto \sqrt{a} b \sqrt{a}$ for positive $a$ and $b$ in a $C^{*}$-algebra, one gets a sequential effect algebra. In a follow-up paper [52] we use the techniques and results of this paper to show that a spectral theorem holds in any normal sequential effect algebra [21] and furthermore that any such algebra is isomorphic to the direct sum of a Boolean, convex and a "almost convex" normal sequential effect algebra.

\footnotetext{
${ }^{3}$ In the reconstruction of Ref. [46], almost all the work is done without any assumptions on the scalars, but in the end, the structure of the unit interval is necessary to get to standard quantum theory, which is something which would not be necessary with our approach.
}

\section{Acknowledgments}

JvdW is supported in part by AFOSR grant FA2386-18-1-4028.

\section{References}

[1] Samson Abramsky and Bob Coecke. 2004. A Categorical Semantics of Quantum Protocols. In Proceedings of the 19th Annual IEEE Symposium on Logic in Computer Science, 2004. IEEE, 415-425.

[2] Robin Adams and Bart Jacobs. [n.d.]. State and Effect Logics for Deterministic, Non-deterministic, Probabilistic and Quantum Computation. TYPES 2015 ([n. d.]), 8.

[3] Michael A Arbib and Ernest G Manes. 1980. Partially Additive Categories and Flow-Diagram Semantics. Fournal of Algebra 62, 1 (1980), 203-227.

[4] Jonathan Barrett. 2007. Information Processing in Generalized Probabilistic Theories. Physical Review A 75, 3 (2007), 032304. https: //doi.org/10.1103/PhysRevA.75.032304

[5] Ivan Chajda, Radomir Halaš, and Jan Kühr. 2009. Every Effect Algebra can be Made into a Total Algebra. Algebra universalis 61, 2 (2009), 139. https://doi.org/10.1007/s00012-009-0010-6

[6] Giulio Chiribella, Giacomo Mauro D'Ariano, and Paolo Perinotti. 2011. Informational Derivation of Quantum Theory. Physical Review A 84, 1 (2011), 012311. https://doi.org/10.1103/PhysRevA.84.012311

[7] Kenta Cho. 2019. Effectuses in Categorical Quantum Foundations. Ph.D. Dissertation. Radboud Universiteit Nijmegen. https:/arxiv.org/abs/ 1910.12198.

[8] Kenta Cho, Bart Jacobs, Bas E Westerbaan, and Abraham A Westerbaan. 2015. An Introduction to Effectus Theory. (2015). https://arxiv.org/ abs/1512.05813.

[9] Kenta Cho, Bas Westerbaan, and John van de Wetering. 2020. Dichotomy between deterministic and probabilistic models in countably additive effectus theory. (2020). https://arxiv.org/abs/2003.10245.

[10] Anatolij Dvurečenskij. 2010. Every State on Interval Effect Algebra is Integral. f. Math. Phys. 51, 8 (2010), 083508. https://doi.org/10.1063/1. 3467463

[11] Pau Enrique Moliner, Chris Heunen, and Sean Tull. 2018. Space in Monoidal Categories. In Proceedings 14th International Conference on Quantum Physics and Logic, Nijmegen, The Netherlands, 3-7 July 2017 (Electronic Proceedings in Theoretical Computer Science), Bob Coecke and Aleks Kissinger (Eds.), Vol. 266. Open Publishing Association, 399-410. https://doi.org/10.4204/EPTCS.266.25

[12] David J Foulis and Mary K Bennett. 1994. Effect Algebras and Unsharp Quantum Logics. Foundations of physics 24, 10 (1994), 1331-1352. https://doi.org/10.1007/BF02283036

[13] David J Foulis and Sylvia Pulmannová. 2010. Type-Decomposition of an Effect Algebra. Foundations of Physics 40, 9-10 (2010), 1543-1565. https://doi.org/10.1007/s10701-009-9344-3

[14] Robert Furber, Dexter Kozen, Kim Larsen, Radu Mardare, and Prakash Panangaden. 2017. Unrestricted Stone Duality for Markov Processes. In 2017 32nd Annual ACM/IEEE Symposium on Logic in Computer Science (LICS). IEEE, 1-9.

[15] Leonard Gillman and Meyer Jerison. 2013. Rings of continuous functions. Springer. https://doi.org/10.1007/978-1-4615-7819-2

[16] Zahra Eslami Giski and Abolfazl Ebrahimzadeh. 2017. An Introduction of Logical Entropy on Sequential Effect Algebra. Indagationes Mathematicae 28, 5 (2017), 928-937. https://doi.org/10.1016/j.indag. 2017.06.007

[17] Stefano Gogioso. 2017. Fantastic Quantum Theories and Where to Find Them. (2017). https://arxiv.org/abs/1703.10576.

[18] Stanley Gudder. 1996. Examples, Problems, and Results in Effect Algebras. International fournal of Theoretical Physics 35, 11 (1996), 23652376. https://doi.org/10.1007/bf02302453

[19] Stan Gudder. 2005. Open Problems for Sequential Effect Algebras. International fournal of Theoretical Physics 44, 12 (2005), 2199-2206. 
https://doi.org/10.1007/s10773-005-8015-1

[20] Stan Gudder. 2008. Sequential products of quantum subtests. Reports on Mathematical Physics 62, 2 (2008), 255-272.

[21] Stan Gudder and Richard Greechie. 2002. Sequential Products on Effect Algebras. Reports on Mathematical Physics 49, 1 (2002), 87-111. https://doi.org/10.1016/S0034-4877(02)80007-6

[22] Stanley Gudder and Sylvia Pulmannová. 1998. Representation theorem for convex effect algebras. Commentationes Mathematicae Universitatis Carolinae 39, 4 (1998), 645-660.

[23] Stanley P Gudder and Richard Greechie. 1996. Effect Algebra Counterexamples. Mathematica Slovaca 46, 4 (1996), 317-325.

[24] Eissa D Habil. 2008. Tensor Product of Distributive Sequential Effect Algebras and Product Effect Algebras. International fournal of Theoretical Physics 47, 1 (2008), 280-290. https://doi.org/10.1007/s10773007-9472-5

[25] John Harding. 2004. Remarks on Concrete Orthomodular Lattices. International fournal of Theoretical Physics 43, 10 (2004), 2149-2168.

[26] Lucien Hardy. 2001. Quantum Theory From Five Reasonable Axioms. (2001). https://arxiv.org/abs/quant-ph/0101012.

[27] Bart Jacobs. 2011. Probabilities, Distribution Monads, and Convex Categories. Theoretical Computer Science 412, 28 (2011), 3323-3336. https://doi.org/10.1016/j.tcs.2011.04.005

[28] Bart Jacobs. 2012. New directions in categorical logic, for classical, probabilistic and quantum logic. arXiv preprint arXiv:1205.3940 (2012).

[29] Bart Jacobs and Jorik Mandemaker. 2012. Coreflections in Algebraic Quantum Logic. Foundations of physics 42, 7 (2012), 932-958. https: //doi.org/10.1007/s10701-012-9654-8

[30] Bart Jacobs and Jorik Mandemaker. 2016. Relating operator spaces via adjunctions. Logic and Algebraic Structures in Quantum Computing 45 (2016), 123-150.

[31] Bart Jacobs and Abraham A Westerbaan. 2015. An Effect-Theoretic Account of Lebesgue Integration. Electronic Notes in Theoretical Computer Science 319 (2015), 239-253. https://doi.org/10.1016/j.entcs.2015.12. 015

[32] Gejza Jenča. 2001. Blocks of Homogeneous Effect Algebras. Bulletin of the Australian Mathematical Society 64, 1 (2001), 81-98. https: //doi.org/10.1017/s0004972700019705

[33] Gejza Jenča. 2015. Effect Algebras are the Eilenberg-Moore Category for the Kalmbach Monad. Order 32, 3 (2015), 439-448.

[34] Peter T Johnstone. 1982. Stone spaces. Vol. 3. Cambridge university press.

[35] Younesse Kaddar and Richard Garner. 2019. Tricocycloids, Effect Monoids and Effectuses. (2019).

[36] Richard V Kadison. 1951. A representation theory for commutative topological algebra. Number 7. American Mathematical Society. https: //doi.org/10.1090/memo/0007

[37] Gudrun Kalmbach. 1977. Orthomodular Lattices Do Not Satisfy Any Special Lattice Equation. Archiv der Mathematik 28, 1 (1977), 7-8

[38] René Mayet and Mirko Navara. 1995. Classes of Logics Representable as Kernels of Measures. Contributions to General Algebra 9 (1995), 241-248.

[39] Sylvia Pulmannová. 2006. Effect algebras with compressions. Reports on Mathematical Physics 58, 2 (2006), 301-324.

[40] Kuppusamy Ravindran. 1996. On a Structure Theory of Effect Algebras. Ph.D. Dissertation. Kansas State University.

[41] Zdenka Riečanová. 2000. Generalization of Blocks for D-lattices and Lattice-Ordered Effect Algebras. International fournal of Theoretical Physics 39, 2 (2000), 231-237.

[42] Frank Roumen. 2016. Cohomology of effect algebras. In QPL 2016 (EPTCS), Vol. 236. 174-202. https://doi.org/10.4204/eptcs.236.12 https: //arxiv.org/abs/1602.00567.

[43] Jun Shen and Junde Wu. 2009. Not Each Sequential Effect Algebra is Sharply Dominating. Physics Letters A 373, 20 (2009), 1708-1712. https://doi.org/10.1016/j.physleta.2009.02.073
[44] Sam Staton and Sander Uijlen. 2018. Effect Algebras, Presheaves, NonLocality and Contextuality. Information and Computation 261 (2018), 336-354. https://doi.org/10.1016/j.ic.2018.02.012

[45] Josef Tkadlec. 2008. Atomic Sequential Effect Algebras. International fournal of Theoretical Physics 47, 1 (2008), 185-192. https://doi.org/10. 1007/s10773-007-9492-1

[46] Sean Tull. 2016. A Categorical Reconstruction of Quantum Theory. (2016). https://arxiv.org/abs/1804.02265.

[47] John van de Wetering. 2019. An effect-theoretic reconstruction of quantum theory. Compositionality 1 (12 2019), 1. https://doi.org/10. 32408/compositionality-1-1

[48] John van de Wetering. 2019. Sequential Product Spaces are Jordan Algebras. J. Math. Phys. 60, 6 (2019), 062201. https://doi.org/10.1063/ 1.5093504

[49] Arnoud C M van Rooij. 2011. Lecture notes on Riesz Spaces. https: //github.com/awesterb/riesz-spaces.

[50] Liu Weihua and Wu Junde. 2009. A Uniqueness Problem of the Sequence Product on Operator Effect Algebra E(H). Journal of Physics A: Mathematical and Theoretical 42, 18 (2009), 185206. https://doi.org/10. 1088/1751-8113/42/18/185206

[51] Abraham A Westerbaan. 2019. The Category of Von Neumann Algebras. Ph.D. Dissertation. Radboud Universiteit Nijmegen. https://doi.org/ 2066/201611 https://arxiv.org/abs/1804.02203.

[52] Abraham A Westerbaan, Bas E Westerbaan, and John van de Wetering. 2019. The three types of normal sequential effect algebras. (2019). https://arxiv.org/abs/2004.12749.

[53] Bas E Westerbaan. 2013. Sequential Product on Effect Logics. Master's thesis. Radboud University Nijmegen. https://www.ru.nl/publish/ pages/813276/masterscriptie bas westerbaan.pdf.

[54] Bas E Westerbaan. 2016. Yosida Duality. (2016). https://arxiv.org/abs/ 1612.03327.

[55] Bas E Westerbaan. 2019. Dagger and Dilations in the Category of von Neumann Algebras. Ph.D. Dissertation. Radboud Universiteit Nijmegen. https://doi.org/2066/201785 https://arxiv.org/abs/1803.01911.

[56] JD Maitland Wright. 1972. Measures with Values in a Partially Ordered Vector Space. Proceedings of the London Mathematical Society 3, 4 (1972), 675-688

[57] Kôsaku Yosida. 1942. On the Representation of the Vector Lattice. Proceedings of the Imperial Academy 18, 7 (1942), 339-342.

[58] Octavio Baltasar Zapata Fonseca. 2019. Logical Aspects of Probability and Quantum Computation. Ph.D. Dissertation. UCL (University College London). 